\title{
Eremophilane Sesquiterpenes From Capsidiol
}

Yuxin Zhao, ${ }^{a}$ David J. Schenk, ${ }^{a}$ Shunji Takahashi, ${ }^{b}$ Joe Chappell, ${ }^{b}$ and Robert M. Coates ${ }^{a}$ *

${ }^{\mathrm{a}}$ Department of Chemistry, University of Illinois, 600 South Mathews Avenue, Urbana, Il 61801

${ }^{\mathrm{b}}$ Department of Agronomy, Plant Physiology, Biochemistry, and Molecular Biology Program, University of Kentucky, Lexington, KY 40546-0091.

\section{SUPPORTING INFORMATION}

Table of Contents

1) Experimental Procedures......................2-15

2) ${ }^{1}$ H NMR Spectra of Selected Compounds........16-37 


\section{General Aspects:}

${ }^{1} \mathrm{H}$ and ${ }^{13} \mathrm{C}$ NMR spectra were recorded in $\mathrm{CDCl}_{3}\left({ }^{1} \mathrm{H}, 7.26 ;{ }^{13} \mathrm{C}, 77.0\right)$ or benzene- $\mathrm{d}_{6}\left({ }^{1} \mathrm{H}, 7.16 ;{ }^{13} \mathrm{C}, 128\right)$ with U400 and U500 spectrometers at the University of Illinois. ${ }^{31} \mathrm{P}$ NMR spectra were externally referenced to $0 \mathrm{ppm}$ with $85 \% \mathrm{H}_{3} \mathrm{PO}_{4}$. Chemical shifts are in ppm and coupling constants are in Hertz. The abbreviation 'app' is used to describe the apparent multiplicity of the peak and may or may not be a valid first-order analysis. Mass spectra were recorded on 70V-SE instruments. Infrared (IR) spectra were obtained using a Spectrum BX spectrophotometer referenced to polystyrene standard. Data are presented as frequency of absorption $\left(\mathrm{cm}^{-1}\right)$. Optical rotations were acquired on a Digital Polarimeter. Melting points were recorded with a melting apparatus and are uncorrected. GC analyses were carried out on a GC with an $\mathrm{Rt}_{\mathrm{x}}-5$ 30-m fused silica capillary column. Programs 1 and 2 follow: (1) initial temperature $60{ }^{\circ} \mathrm{C}$, hold for $7 \mathrm{~min}, 10{ }^{\circ} \mathrm{C} / \mathrm{min}$ until $275^{\circ} \mathrm{C}$, hold for $20 \mathrm{~min}$; (2) initial temperature $35^{\circ} \mathrm{C}$, hold for $7 \mathrm{~min}, 5$ ${ }^{\circ} \mathrm{C} / \min$ until $275^{\circ} \mathrm{C}$.

All chemical reactions were performed in flame-dried glassware under nitrogen. THF and $\mathrm{Et}_{2} \mathrm{O}$ were dried and distilled from $\mathrm{Na}$ /benzophenone; Benzene and $\mathrm{CH}_{2} \mathrm{Cl}_{2}$ were dried and distilled from $\mathrm{CaH}_{2}$. Hexane and ethyl acetate were freshly distilled from $\mathrm{CaH}_{2}$. TLC analyses were performed on silica gel 60 F254 precoated-plates $250 \mu \mathrm{m}$. All retention factors $\left(\mathrm{R}_{\mathrm{f}}\right)$ are on silica gel TLC plates until otherwise noted. TLC visualizations were performed with $5 \%$ phosphomolybdic acid $(0.2 \mathrm{M}$ in $2.5 \%$ conc. $\left.\mathrm{H}_{2} \mathrm{SO}_{4} / \mathrm{EtOH}(\mathrm{v} / \mathrm{v})\right)$, $\mathrm{I}_{2}$, or UV. Commercial reagents were used without further purification unless specifically noted. Column chromatography was performed according to Still's procedure ${ }^{1}$ using $100-700$ times excess 32-64 $\mu \mathrm{m}$ grade silica gel. Products separated by chromatography are specified in elution order. In some cases the yields of products containing solvents were corrected for the solvent peak integration in ${ }^{1} \mathrm{H}$ NMR spectra and specified individually in the data sections. $\mathrm{AgNO}_{3}$-impregnated silica gel $(15 \%, w / w)$ was prepared by adding silica gel $(170 \mathrm{~g})$ to a solution of $\mathrm{AgNO}_{3}(30 \mathrm{~g})$ in freshly distilled acetonitrile $(400 \mathrm{~mL})$, which was mixed well with a glass rod. Most of the solvent was removed by rotavap, and then the remainder was removed at high vacuum $(1 \mathrm{~mm} \mathrm{Hg})$ until the weight was about 200g. The $\mathrm{AgNO}_{3}$-silica gel was stored under nitrogen in the dark. ${ }^{2}$ HPLC separations were performed using a UV Detector with a semi-preparative scale column.

Green peppers were purchased locally and used the same day or were refrigerated until use. Deionized (DI) water was used for elicitations. Cellulase (Karlan Cellulase RS, Tricoderma viride) was stored in a 4 ${ }^{\circ} \mathrm{C}$ refrigerator when not being used.

1 Still, W. C.; Kahn, M.; Mitra, A. J. Org. Chem. 1978, 43, 2923.

2 Yee, N. K. N.; Coates, R. M. J. Org. Chem. 1992, 57, 4598. 


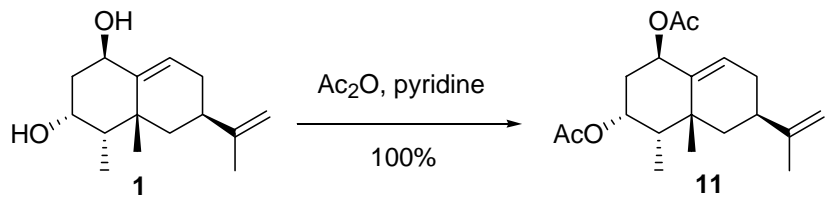

Capsidiol Diacetate (11) The modified procedure of Stothers ${ }^{3}$ was followed. A solution of capsidiol (40 $\mathrm{mg}, 0.17 \mathrm{mmol})$ in acetic anhydride $(4.6 \mathrm{~g}, 4.3 \mathrm{~mL}, 45 \mathrm{mmol})$ was stirred and cooled at $0{ }^{\circ} \mathrm{C}$ as pyridine $(6.8 \mathrm{~g}, 7 \mathrm{~mL}, 86 \mathrm{mmol})$ was added slowly over $2-3 \mathrm{~min}$. The reaction mixture was warmed up to $\mathrm{rt}$ and stirred for $16 \mathrm{~h}$. Satd. $\mathrm{NaHCO}_{3}(20 \mathrm{~mL})$ and ether $(20 \mathrm{~mL})$ were added, the aqueous layer was extracted with ether $(4 \times 20 \mathrm{~mL})$, and the combined organic layers were washed with $1 \% \mathrm{HCl}(15 \mathrm{~mL}), \mathrm{Cu}\left(\mathrm{NO}_{3}\right)_{2}$ (20x $2 \mathrm{~mL})$ and satd. $\mathrm{NaCl}(1 \times 20 \mathrm{~mL})$, and dried $\left(\mathrm{MgSO}_{4}\right)$. Purification by chromatography $(6: 1$ hexane/ethyl acetate) afforded $55 \mathrm{mg}(100 \%)$ of the known capsidiol diacetate ${ }^{1,2}$ as colorless oil. The purity was shown to be $>95 \%$ by ${ }^{1} \mathrm{H}$ NMR analysis. The following data are similar to those in the literature ${ }^{4}$ : TLC $\mathrm{R}_{\mathrm{f}} 0.31$ (6:1 hexane/ethyl acetate); ${ }^{1} \mathrm{H}$ NMR $\left(400 \mathrm{MHz}, \mathrm{CDCl}_{3}\right) \delta 6.10$ (dd, $1 \mathrm{H}, J=6.7$, $2.1 \mathrm{~Hz},=\mathrm{CH}), 5.54(\mathrm{dt}, 1 \mathrm{H}, J=12.8,4.4 \mathrm{~Hz}, \mathrm{CHOAc}), 5.41(\mathrm{dd}, 1 \mathrm{H}, J=3.8,2.8 \mathrm{~Hz}, \mathrm{CHOAc}), 4.71$ (t, $\left.1 \mathrm{H}, J=1.2 \mathrm{~Hz},=\mathrm{CH}_{2}\right), 4.67\left(\mathrm{~s}, 1 \mathrm{H},=\mathrm{CH}_{2}\right), 2.12\left(\mathrm{~m}, 2 \mathrm{H}, \mathrm{CH}\right.$ or $\left.\mathrm{CH}_{2}\right), 2.05\left(\mathrm{~s}, 3 \mathrm{H}, \mathrm{CH}_{3}\right), 2.02(\mathrm{~s}, 3 \mathrm{H}$, $\left.\mathrm{CH}_{3}\right), 1.91\left(\mathrm{~m}, 3 \mathrm{H}, \mathrm{CH}\right.$ and $\left.\mathrm{CH}_{2}\right), 1.80\left(\mathrm{~m}, 2 \mathrm{H}, \mathrm{CH}_{2}\right), 1.72\left(\mathrm{~s}, 3 \mathrm{H},=\mathrm{CCH}_{3}\right), 1.32\left(\mathrm{~m}, 1 \mathrm{H}, \mathrm{CH}_{2}, \mathrm{H} 6 \beta\right), 1.32$ (s, $\left.3 \mathrm{H}, \mathrm{CH}_{3}\right), 0.88\left(\mathrm{~d}, 3 \mathrm{H}, J=7.2 \mathrm{~Hz}, \mathrm{CH}_{3}\right)$.

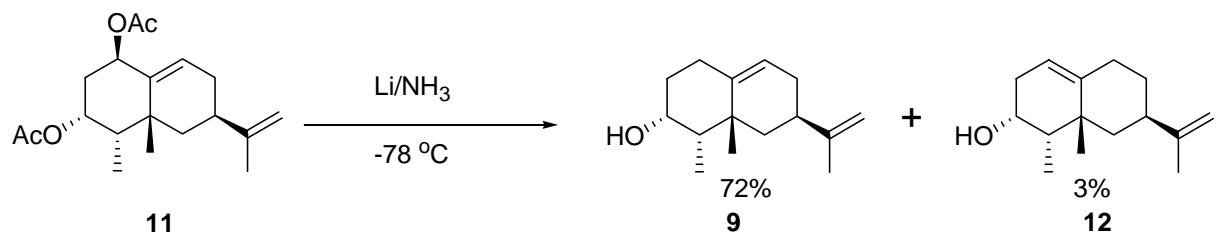

1-Deoxycapsidiol (9) and Epieremophilene-3 $\alpha-0$ l (12) The modified procedure of Whitehead ${ }^{5}$ was followed. $\mathrm{NH}_{3}$ gas was condensed into a flame-dried, 3-necked flask under $\mathrm{N}_{2}$ in a $-78{ }^{\circ} \mathrm{C}$ bath (acetone/dry ice). Pieces of $\mathrm{Li}$ rod $\left(20 \mathrm{mg}, 2.9 \mathrm{mmol}\right.$ ) were added to the liquid $\mathrm{NH}_{3}(25 \mathrm{~mL})$ and the suspension was stirred for $20 \mathrm{~min}$ until the color turned to deep blue. Capsidiol diacetate (11) (0.055 g, $0.156 \mathrm{mmol})$ dissolved in THF $(1.5 \mathrm{~mL})$ was added slowly, and the resulting solution was stirred for 50 min at $-78^{\circ} \mathrm{C}$. $t$-Butyl alcohol $(0.78 \mathrm{~g}, 1.0 \mathrm{~mL}, 10.5 \mathrm{mmol})$ dissolved in THF $(4 \mathrm{~mL})$ was added, and the

3 Hoyano, Y.; Stoessl, A.; Stothers, J. B. Can. J. Chem. 1980, 58, 1894.

4 Gordon, M.; Stoessl, A.; Stothers, J. B. Can. J. Chem. 1974, 52, 993.

5 Whitehead, I. M.; Ewing, D. F.; Threlfall, D. R.; Cane, D. E.; Prabhakaran, P. C. Phytochemistry 1990, 29, 479. 
$\mathrm{NH}_{3}$ was removed by a gentle stream of $\mathrm{N}_{2}$. The aqueous layer was extracted with ether $(3 \times 20 \mathrm{~mL})$ and hexane $(20 \mathrm{~mL})$. The combined organic layers were washed with $\mathrm{HCl}(1 \mathrm{M}, 2 \times 20 \mathrm{~mL})$, brine $(15 \mathrm{~mL})$, dried $\left(\mathrm{MgSO}_{4}\right)$, and concentrated affording $32 \mathrm{mg}$ of crude alcohols. The crude product was determined to be a 12:1 mixture of 1-deoxycapsidiol and epieremophilene-3 $\alpha$-ol by ${ }^{1} \mathrm{H}$ NMR analysis. Purification by chromatography on silica gel (5:1 hexane/ethyl acetate) provided $2 \mathrm{mg}$ (3\%) of epieremophilene $3 \alpha-\mathrm{ol}$ and $25.1 \mathrm{mg}(72 \%)$ of 1-deoxycapsidiol. ${ }^{1} \mathrm{H}$ NMR data were consistant to those previously reported by Whitehead et al. ${ }^{5}$ Data for epieremophilene $3 \alpha$-ol (12): TLC R 0.28 (4:1 hexane/ethyl acetate); $[\alpha]_{\mathrm{D}}=$ $1.4 \times 10^{2} \pm 10\left(\mathrm{c}=0.13, \mathrm{CHCl}_{3}\right) ;{ }^{1} \mathrm{H} \mathrm{NMR}\left(500 \mathrm{MHz}, \mathrm{CDCl}_{3}\right) \delta 5.22(\mathrm{dt}, 1 \mathrm{H}, J=4.5,2.5,=\mathrm{CH}), 4.72(\mathrm{~d}$, $\left.2 \mathrm{H}, J=4.9 \mathrm{~Hz},=\mathrm{CH}_{2}\right), 4.18\left(\mathrm{ddd}, 1 \mathrm{H}, J=9.8,5.8,3.5, \mathrm{CH}_{2} \mathrm{CHOH}\right), 2.39(\mathrm{dtt}, 1 \mathrm{H}, J=14.0,9.0,2.6 \mathrm{~Hz})$, $2.21(\mathrm{ddt}, J=17.0,9.5,2.9 \mathrm{~Hz}), 2.14(\mathrm{~m}, 1 \mathrm{H}), 2.09-1.93(\mathrm{~m}, 2 \mathrm{H}), 1.80(\mathrm{dd}, 1 \mathrm{H}, J=13.5,4.0 \mathrm{~Hz}), 1.73$ $\left(\mathrm{dd}, J=1.2,0.6 \mathrm{~Hz}, \mathrm{CH}_{3}\right), 1.72-1.61(\mathrm{~m}, 3 \mathrm{H}), 1.40$ (dd, $\left.J=13.8,9.8 \mathrm{~Hz}, \mathrm{H} 6 \beta\right), 1.18\left(\mathrm{~s}, 3 \mathrm{H}, \mathrm{CH}_{3}\right), 0.93$ $\left(\mathrm{d}, 3 \mathrm{H}, J=7.0, \mathrm{CH}_{3}\right.$ ). The ${ }^{1} \mathrm{H}$ NMR data for 1-deoxycapsidiol (9) are consistant to those reported by Whitehead et al. ${ }^{4}$ : TLC $\mathrm{R}_{\mathrm{f}} 0.27$ (4:1 hexane/ethyl acetate); ${ }^{1} \mathrm{H}$ NMR $\left(500 \mathrm{MHz}, \mathrm{CDCl}_{3}\right) \delta 5.64(\mathrm{dt}, 1 \mathrm{H}, J$ = 7.0, $1.5 \mathrm{~Hz},=\mathrm{CH}), 4.70(\mathrm{t}, 1 \mathrm{H}, J=1.5 \mathrm{~Hz},=\mathrm{CH}), 4.68(\mathrm{~m}, 1 \mathrm{H},=\mathrm{CH}), 4.21(\mathrm{dt}, 1 \mathrm{H}, J=12.0,4.7 \mathrm{~Hz}$, $\mathrm{CHOH}), 2.32(\mathrm{bt}, 1 \mathrm{H}, J=14 \mathrm{~Hz}, \mathrm{H} 7), 2.26$ (tt, 1H, $J=12.5,3.7 \mathrm{~Hz}, \mathrm{H} 1 \alpha), 2.09 \sim 2.02$ (m, 2H, H1 $\alpha$ and $\mathrm{H} 8 \alpha$ ), 1.80 (ddq, $1 \mathrm{H}, J=16.0,11.5,1.5 \mathrm{~Hz}, \mathrm{H} 8 \beta), 1.76$ (dt, 1H, J=14.0, 3.0 Hz, $\left.\mathrm{CH}_{2}\right), 1.73\left(\mathrm{~s}, 3 \mathrm{H}, \mathrm{CH}_{3}\right)$, $1.68\left(\mathrm{~m}, 2 \mathrm{H}, \mathrm{CH}_{2}\right), 1.41(\mathrm{~m}, 2 \mathrm{H}, 2 \mathrm{CH}), 1.29\left(\mathrm{t}, 1 \mathrm{H}, J=13.5 \mathrm{~Hz}, \mathrm{CH}_{2}, \mathrm{H} 6 \beta\right), 1.16\left(\mathrm{~s}, 3 \mathrm{H}, \mathrm{CH}_{3}\right), 0.91$ (d, $\left.3 \mathrm{H}, J=7.0 \mathrm{~Hz}, \mathrm{CH}_{3}\right)$.
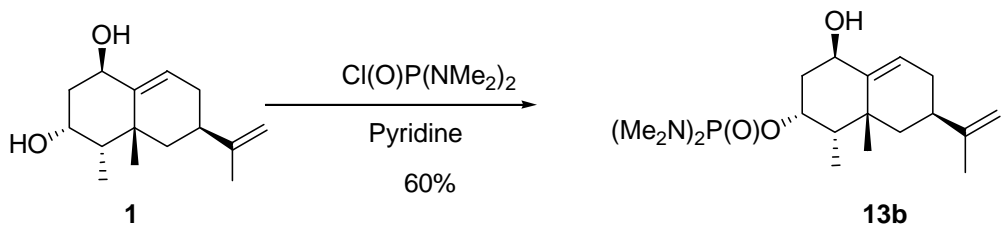

Capsidiol 3-Phosphoramidate (13b) The general method of Ireland ${ }^{6}$ was followed. A solution of capsidiol (1) (30 mg, $0.127 \mathrm{mmol}, 1.0$ equiv) in pyridine $(0.4 \mathrm{~mL})$ was stirred as tetramethylphosphorodiamidic chloride $(100 \mu \mathrm{L}, 0.6 \mathrm{mmol}, 5$ equiv) was added over $2-3 \mathrm{~min}$. The solution was then stirred for $18 \mathrm{~h}$, and the reaction mixture diluted with $\mathrm{Et}_{2} \mathrm{O}(15 \mathrm{~mL})$ and $\mathrm{H}_{2} \mathrm{O}(5 \mathrm{~mL})$. The aqueous layer was extracted with $\mathrm{Et}_{2} \mathrm{O}(3 \mathrm{x} 25 \mathrm{~mL})$. The combined $\mathrm{Et}_{2} \mathrm{O}$ extracts were washed with satd. $\mathrm{Cu}\left(\mathrm{NO}_{3}\right)_{2}(2 \times 15 \mathrm{~mL}), \mathrm{H}_{2} \mathrm{O}(10 \mathrm{~mL})$, dried $\left(\mathrm{MgSO}_{4}\right)$ and concentrated. Purification of the crude product (40 mg) by chromatography on silica gel (95:5 ethyl acetate/MeOH) provided capsidiol 3-

6 Ireland, R. E.; Muchmore, D. C.; Hengartner, U. J. Am. Chem. Soc. 1972, 94, 5098. 
phosphoramidate (13b) (33 mg, 60\%) as a somewhat unstable white solid and recovered capsidiol (5 mg). Data for 13b: TLC R 0.30 (95:5 ethyl acetate/MeOH); mp 117.5-119 ${ }^{\circ} \mathrm{C} ;{ }^{1} \mathrm{H}$ NMR (400 MHz, $\left.\mathrm{CDCl}_{3}\right) \delta$ $5.92(\mathrm{dd}, 1 \mathrm{H}, J=6.9,2.0 \mathrm{~Hz}), 5.07$ (ddt, 1H, $J=12.6,9.0,4.6 \mathrm{~Hz}), 4.69$ (ddd, 2H, J = 13.5, 1.6, $1.5 \mathrm{~Hz})$, $4.35(\mathrm{dd}, 1 \mathrm{H} J=3.4,2.7 \mathrm{~Hz}), 2.68(\mathrm{~m}, 1 \mathrm{H}), 2.65\left(\mathrm{~d}, 6 \mathrm{H}, J=0.8 \mathrm{~Hz}, \mathrm{NMe}_{2}\right), 2.62(\mathrm{~d}, 6 \mathrm{H}, J=1.0 \mathrm{~Hz}$, $\left.\mathrm{NMe}_{2}\right), 2.16(\mathrm{tt}, 1 \mathrm{H}, J=12.3,2.3 \mathrm{~Hz}), 2.11 \sim 2.01(\mathrm{~m}, 3 \mathrm{H}), 1.88(\mathrm{qd}, 1 \mathrm{H}, J=11.6,2.1 \mathrm{~Hz}), 1.79(\mathrm{tt}, 2 \mathrm{H}, J$ $=15.7,3.0 \mathrm{~Hz}), 1.72(\mathrm{~s}, 3 \mathrm{H}) 1.40(\mathrm{~s}, 3 \mathrm{H}), 1.31(\mathrm{dd}, 1 \mathrm{H}, J=13.8,13.2 \mathrm{~Hz}, \mathrm{H} 6 \beta), 0.91(\mathrm{~d}, 3 \mathrm{H}, J=17.1$ $\mathrm{Hz}) ;{ }^{13} \mathrm{C} \mathrm{NMR}\left(100 \mathrm{MHz}, \mathrm{CDCl}_{3}\right) \delta 149.4,139.7,128.9,108.7,74.5,70.7,70.7,46.6,46.6,44.8,40.0$, 36.6, 36.5, 35.1, 35.1, 32.0, 30.2, 21.0, 9.7; ${ }^{31} \mathrm{P} \mathrm{NMR}\left(400 \mathrm{MHz}, \mathrm{CDCl}_{3}\right) \delta 19.8$; FTIR (neat film) 3350.5, 2922.1, 1643.5, 1456.5, 1375.2, 1303.2, 1209.1, 1071.7, 982.5, 891.1, $755.8 \mathrm{~cm}^{-1}$; HRMS (FAB) m/z Calcd for $\mathrm{C}_{19} \mathrm{H}_{35} \mathrm{~N}_{2} \mathrm{O}_{3} \mathrm{P}(\mathrm{M}+\mathrm{H})^{+}: 371.2463$, found 371.2463 .
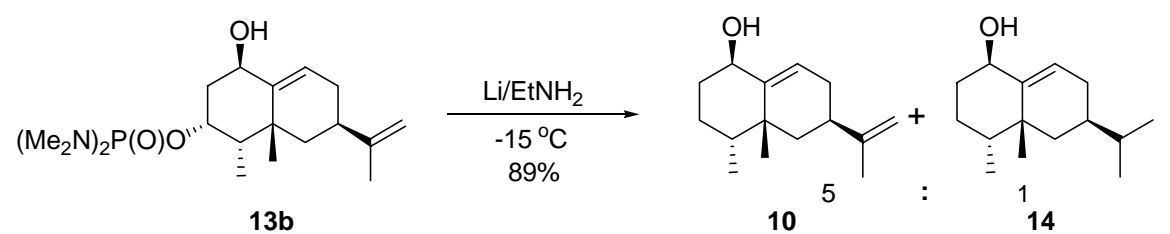

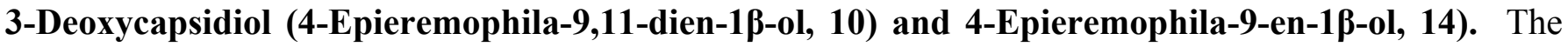
general procedure of Valenta et al. was followed. Ethylamine gas was condensed into a flame-dried, 3necked flask under $\mathrm{N}_{2}$ cooled at $-78^{\circ} \mathrm{C}$ bath (acetone/dry ice). Pieces of Li rod (20 mg, $2.9 \mathrm{mmol}$ ) were added to the liquid ethylamine $(25 \mathrm{~mL})$. The bath was changed to a $0{ }^{\circ} \mathrm{C}$ bath, and the suspension was stirred for $20 \mathrm{~min}$ until its color turned to deep blue. The dried ethylamine $(15 \mathrm{ml})$ was transferred by distillation into a second flame-dried flask under $\mathrm{N}_{2}$ and cooled to $-78{ }^{\circ} \mathrm{C}$. Li pieces $(20 \mathrm{mg}, 2.9 \mathrm{mmol})$ were added, and the bath was replaced by a $-15^{\circ} \mathrm{C}$ one (methanol/ice). Capsidiol 3-phosphoramidate (13b) (50 mg, $0.13 \mathrm{mmol}$ ) in $0.8 \mathrm{~mL}$ of dry THF was added by drops after the color of the solution turned into deep blue. After $15 \mathrm{~min}$, tert-butyl alcohol $(30 \mu \mathrm{L}, 0.28 \mathrm{mmol})$ in $0.5 \mathrm{~mL}$ THF was added. After 5 min, 3-hexyne $(72 \mathrm{mg}, 0.1 \mathrm{~mL}, 0.9 \mathrm{mmol})$, satd $\mathrm{NH}_{4} \mathrm{Cl}$ solution $(2 \mathrm{~mL})$, and ethyl ether $(15 \mathrm{~mL})$ were added sequentially. The mixture was warmed to room temperature, and ethylamine was removed by a gentle $\mathrm{N}_{2}$ stream. The product was extracted with $\mathrm{Et}_{2} \mathrm{O}(3 \times 15 \mathrm{~mL})$. The combined ether extracts $(60 \mathrm{~mL})$ were washed with $10 \% \mathrm{HCl}(20 \mathrm{~mL})$ and brine $(15 \mathrm{~mL})$, dried $\left(\mathrm{MgSO}_{4}\right)$, and concentrated. Purification of the crude product $(30 \mathrm{mg})$ by chromatography on silica gel (4:1 hexane/ethyl acetate) provided $27.2 \mathrm{mg}$ $(89 \%)$ of a 5:1 mixture of 3-deoxycapsidiol (10) and 4-epieremorphila-9-en-1 $\beta$-ol (14) according to GC analysis (Program 1). Further purification by preparative HPLC (6:1 hexane/ethyl acetate) afforded pure samples of 3-deoxycapsidiol (11 mg) and 4-epieremorphila-9-en-1 $\beta$-ol (5 mg) as pale yellow oils. Data for 4-epieremophila-9-en-1 $\beta$-ol (14): $\mathrm{TLC} \mathrm{R}_{\mathrm{f}} 0.22$ (6:1 hexane/ethyl acetate); HPLC $\mathrm{t}_{R}$ (11 min, 16 
$\mathrm{mL} / \mathrm{min}, 6: 1$ hexane/ethyl acetate); ${ }^{1} \mathrm{H} \mathrm{NMR}\left(500 \mathrm{MHz}, \mathrm{CDCl}_{3}\right) \delta 5.83(\mathrm{dd}, 1 \mathrm{H}, J=6.9,1.6 \mathrm{~Hz}), 4.23$ (s, 1H), $2.33(\mathrm{tt}, 1 \mathrm{H}, J=13.7,4.7 \mathrm{~Hz}), 2.01(\mathrm{dqt}, 1 \mathrm{H}, J=16.4,3.4 \mathrm{~Hz}), 1.73(\mathrm{tt}, 1 \mathrm{H}, J=14.5,4.1 \mathrm{~Hz}), 1.66$ (ddt, $2 \mathrm{H}, J=14.3,6.2,3.0 \mathrm{~Hz}), 1.57(\mathrm{td}, 1 \mathrm{H}, J=11.4,1.9 \mathrm{~Hz}), 1.34(\mathrm{~m} 1 \mathrm{H}), 1.33(\mathrm{~s}, 3 \mathrm{H}), 1.28(\mathrm{~s}, 1 \mathrm{H})$, $1.27(\mathrm{~m}, 1 \mathrm{H}), 1.17$ (dqt, 1H, $J=13.8,2.4 \mathrm{~Hz}$ ), 1.07 (app t, 1H, $J=13.2 \mathrm{~Hz}, \mathrm{H} 6 \beta$ ), 0.90 (overlapping d, $\left.6 \mathrm{H}, J=7.1 \mathrm{~Hz}, \mathrm{CH}\left(\mathrm{CH}_{3}\right)_{2}\right), 0.87(\mathrm{~d}, 3 \mathrm{H}, J=6.5 \mathrm{~Hz}) ;{ }^{13} \mathrm{C} \mathrm{NMR}\left(126 \mathrm{MHz}, \mathrm{CDCl}_{3}\right) \delta 142.0,127.7,74.9$, 43.2, 41.3, 39.3, 38.1, 32.9, 32.7, 29.1, 28.0, 24.9, 20.0, 19.9, 16.8; FTIR (neat film) 3368.8, 2955.6, 2972.5, 2866.9, 1460.1, 1368.0, 1031.6, 1010.7, $908.4 \mathrm{~cm}^{-1}$; HRMS (EI) m/z Calcd for $\mathrm{C}_{15} \mathrm{H}_{26} \mathrm{O} \mathrm{M}^{+}$: 222.1985, found 222.1985. The data for 3-deoxycapsidiol are identical to those presented in the experimental section.
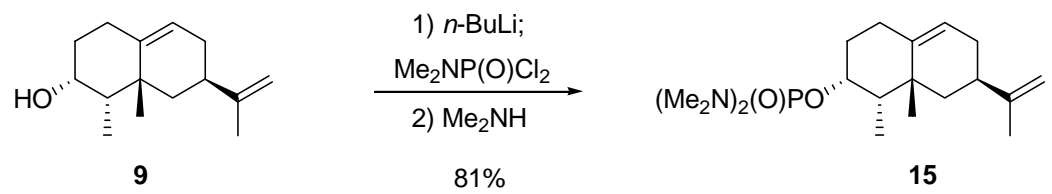

1-Deoxycapsidiol Phosphoramidate (15) The general procedure of Valenta ${ }^{7}$ was followed. A solution of 1-deoxycapsidiol (9) (20 mg, $0.091 \mathrm{mmol})$ and TMEDA (250 mg, $0.327 \mathrm{~mL}, 2.17 \mathrm{mmol})$ in DME (0.7 $\mathrm{mL})$ was stirred and cooled at $0{ }^{\circ} \mathrm{C}$ as $n$-BuLi (1.6 M in hexane, $\left.130 \mu \mathrm{L}, 0.21 \mathrm{mmol}\right)$ was added slowly. The reaction mixture was allowed to stir for $30 \mathrm{~min}$ and $\mathrm{Cl}_{2} \mathrm{P}(\mathrm{O}) \mathrm{NMe}_{2}(320 \mathrm{mg}, 0.4 \mathrm{~mL}, 0.2 \mathrm{mmol})$ was added. The solution was warmed up to rt, stirred for $3 \mathrm{~h}$, and cooled to $-30{ }^{\circ} \mathrm{C}$ after which $\mathrm{Me}_{2} \mathrm{NH}(8.9 \mathrm{~g}$, $10 \mathrm{~mL}, 197 \mathrm{mmol}$ ) was added. The solution was warmed up to $0{ }^{\circ} \mathrm{C}$, stirred for another $2 \mathrm{~h}$, and poured onto ice $(15 \mathrm{~g})$. Ether $(20 \mathrm{~mL})$ was added and the aqueous layer was extracted with ether $(4 \mathrm{x} 25 \mathrm{~mL})$. The combined organic layers were washed with $\mathrm{H}_{2} \mathrm{O}(2 \mathrm{x} 20 \mathrm{~mL})$, dried $\left(\mathrm{MgSO}_{4}\right)$, and concentrated. Chromatography on silica gel $(3 \% \mathrm{MeOH}$ in ethyl acetate) afforded $24.0 \mathrm{mg}(81 \%)$ of the phosphoramidate as a clear oil. ${ }^{1} \mathrm{H}$ NMR analysis determined that the purity of $\mathbf{1 5}$ was $>95 \%$ with residual solvent peaks of ethyl acetate. TLC $\mathrm{R}_{\mathrm{f}} 0.40\left(2 \% \mathrm{MeOH}\right.$ in ethyl acetate); ${ }^{1} \mathrm{H} \mathrm{NMR}\left(500 \mathrm{MHz}, \mathrm{CDCl}_{3}\right) \delta$ $5.59(\mathrm{~d}, 1 \mathrm{H}, J=7.0 \mathrm{~Hz},=\mathrm{CH}), 4.76(\mathrm{ddt}, 1 \mathrm{H}, J=12.3,7.7,4.7 \mathrm{~Hz}, \mathrm{CHOP}), 4.70\left(\mathrm{~s}, 1 \mathrm{H},=\mathrm{CH}_{2}\right), 4.67(\mathrm{~s}$, $\left.1 \mathrm{H},=\mathrm{CH}_{2}\right), 2.61\left(\mathrm{~d}, 6 \mathrm{H}, J=4.0 \mathrm{~Hz}, \mathrm{NMe}_{2}\right), 2.59\left(\mathrm{~d}, 6 \mathrm{H}, J=4.1 \mathrm{~Hz}, \mathrm{NMe}_{2}\right), 2.34(\mathrm{tt}, 1 \mathrm{H}, J=14.0,5.5 \mathrm{~Hz}$, $\mathrm{CH}_{2}$ ), 2.25 (tt, $1 \mathrm{H}, J=12.3,3.5 \mathrm{~Hz}, \mathrm{CH}_{2}$ ), 2.03 (d of sextets, $\left.2 \mathrm{H}, J=14.5,4.5 \mathrm{~Hz}, \mathrm{CH}_{2}\right), 1.80(\mathrm{~m}, 4 \mathrm{H}, \mathrm{CH}$ or $\left.\mathrm{CH}_{2}\right), 1.73\left(\mathrm{~s}, 3 \mathrm{H}, \mathrm{CH}_{3}\right), 1.55\left(\mathrm{dtd}, 1 \mathrm{H}, J=14.0,12.2,5.2 \mathrm{~Hz}, \mathrm{CH}_{2}\right), 1.29($ app t, $1 \mathrm{H}, J=13.3 \mathrm{~Hz}$, $\mathrm{H} 6 \beta), 1.18\left(\mathrm{~s}, 3 \mathrm{H}, \mathrm{CH}_{3}\right), 0.95\left(\mathrm{~d}, 3 \mathrm{H}, J=7.5 \mathrm{~Hz}, \mathrm{CH}_{3}\right) .{ }^{13} \mathrm{C} \mathrm{NMR}\left(\mathrm{CDCl}_{3}, 125 \mathrm{MHz}\right)$ $\delta 149.8,138.2,122.6,108.4,73.8(\mathrm{~d}, J=5.0 \mathrm{~Hz}), 46.3(\mathrm{~d}, J=4.3 \mathrm{~Hz}), 44.0,39.7,36.5(\mathrm{dd}, J=5.9,3.3$

7 Das, J.; Valenta, Z.; Liu, H-J.; Ngooi, T. K. Can. J. Chem. 1984, 62, 481. 
$\mathrm{Hz}), 30.1,29.7,29.4,28.6(\mathrm{~d}, J=3.4 \mathrm{~Hz}), 21.0,10.1 .{ }^{31} \mathrm{P} \quad \mathrm{NMR}\left(500 \mathrm{MHz}, \mathrm{CDCl}_{3}\right) \delta 19.6 ; \mathrm{HRMS}$ (FAB) $\mathrm{m} / \mathrm{z}$ Calcd for $(\mathrm{M}+\mathrm{H})^{+}: 355.2514$, found 355.2514 .
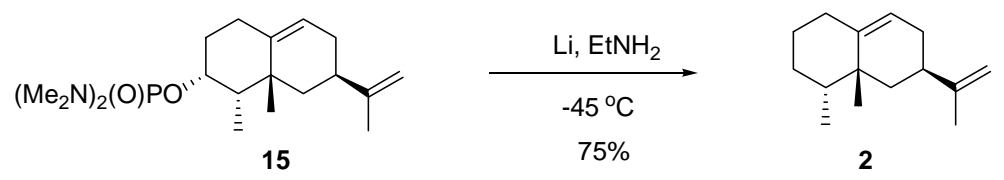

(+)-Epiaristolochene (2). Method A The procedure was the same as the one on page S5 except that the bath temperature changed to $-45^{\circ} \mathrm{C}$. The amounts of reagents were as follow: ethylamine $(25 \mathrm{~mL})$, pieces of Li rod (20 mg, $2.9 \mathrm{mmol}$ ), 1-deoxycapsidiol 3-phosphoramidate (30 mg, $0.085 \mathrm{mmol}$ ), THF (0.8 mL), t-butyl alcohol ( $8 \mu \mathrm{L}$ in $1 \mathrm{~mL}$ of THF, $6 \mathrm{mg}, 0.08 \mathrm{mmol}$ ), and several drops of 3-hexyne. The yield of epiaristolochene was determined to be $18 \mathrm{mg}(86 \%)$ by GC analysis using a cedrene internal standard. Chromatography on silica gel (pentane) afforded epiaristolochene containing residual pentane. The molar ratio of epiaristolochene and pentane was estimated to be $\sim 1: 1.3$ ( ${ }^{1} \mathrm{H}$ NMR), and the purity was $95 \%$ determined by GC analysis (Program 2). The ${ }^{1} \mathrm{H}$ NMR data in $\mathrm{CDCl}_{3}$ are similar to those reported by Whitehead et. al. TLC $\mathrm{R}_{\mathrm{f}} 0.65$ (pentane); ${ }^{1} \mathrm{H}$ NMR $\left(500 \mathrm{MHz}, \mathrm{CDCl}_{3}\right) \delta 5.53$ (dt, $1 \mathrm{H}, J=6.5,1.4 \mathrm{~Hz}, \mathrm{H} 9$ ), 4.69 (s, 1H, H12), 4.67 (s, 1H, H1), 2.24 (m, 1H, H1), 1.99 (m, 1H, $\left.\mathrm{CH}_{2}\right), 1.97$ (m, 1H, $\left.\mathrm{CH}_{2}\right), 1.96$ (m, $1 \mathrm{H}, \mathrm{CH}_{2}$ ), 1.80 (ddq, $\left.1 \mathrm{H}, J=15.7,11.5,2.0 \mathrm{~Hz}, \mathrm{H} 8\right), 1.73$ (s, 3H, $\mathrm{CH}_{3}$ ), 1.68 (dt, $1 \mathrm{H}, J=13.5,3.0, \mathrm{H} 6 \alpha$ ), 1.55 1.42 (m, 3H, $\left.\mathrm{CH}_{2}\right), 1.35 \sim 1.20\left(\mathrm{~m}, 3 \mathrm{H}, \mathrm{CH}\right.$ or $\left.\mathrm{CH}_{2}\right), 1.27\left(\mathrm{~m}, 6 \mathrm{H}\right.$, pentane $\left.\mathrm{CH}_{2}\right), 1.17\left(\mathrm{~s}, 3 \mathrm{H}, \mathrm{CH}_{3}\right)$, 0.98 (d, 3H, $J=7.0, \mathrm{CH}_{3}$ ), 0.88 (t, $6 \mathrm{H}, J=7 \mathrm{~Hz}$, pentane $\mathrm{CH}_{3}$ ); MS (EI) $\mathrm{m} / \mathrm{z}$ 205(3.1), 204(M+1 18.7), 189(11.6), 161(36.6), 147(11.8), 133(20.3), 121(25.4), 119(15.7), 105(100), 93(28.3), 79(17.2), 55(14.6).

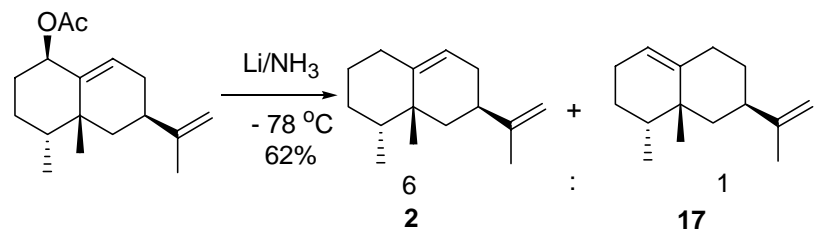

Epiaristolochene (2). Method B The procedure given on p. S3 was followed. The amounts of reagents were as follows: liquid $\mathrm{NH}_{3}(25 \mathrm{~mL})$, pieces of Li rod (20 mg, $\left.2.9 \mathrm{mmol}\right)$, 3-deoxycapsidiol acetate (25 $\mathrm{mg}, 0.01 \mathrm{mmol}$ dissolved in $1.5 \mathrm{~mL}$ of THF), and t-butyl alcohol (78 mg, $0.1 \mathrm{~mL}, 1 \mathrm{mmol}$ in $4 \mathrm{~mL}$ of THF). The crude product (32 mg) was determined to be a 6:1 mixture of epiaristolochene (2) and epieremophilene (17) by GC analysis (program 2). Purification on $15 \%(\mathrm{w} / \mathrm{w}) \mathrm{AgNO}_{3}$ silica gel afforded 
product $(12 \mathrm{mg}, 62 \%)$ as a $6: 1$ mixture of isomers. ${ }^{1} \mathrm{H}$ NMR data for epiaristolochene from the $6: 1$ mixture were consistent with the literature data and the data presented.

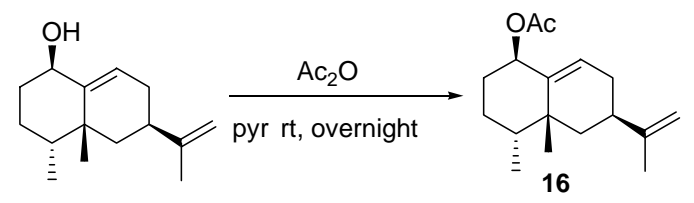

3-Deoxycapsidiol Acetate (16) A solution of 3-deoxycapsidiol (10) (20 mg, $0.09 \mathrm{mmol})$ in acetic anhydride $(0.74 \mathrm{~g}, 0.7 \mathrm{~mL}, 7.3 \mathrm{mmol})$ was stirred and cooled at $0{ }^{\circ} \mathrm{C}$ as pyridine $(0.97 \mathrm{~g}, 1.0 \mathrm{~mL}, 12.3$ mmol) was added slowly over 2-3 min. The reaction mixture was warmed up to rt and stirred overnight. Satd. $\mathrm{NaHCO}_{3}(20 \mathrm{~mL})$ and ether $(20 \mathrm{~mL})$ were added, the aqueous layer was extracted with ether $(4 \mathrm{x} 20$ $\mathrm{mL})$, and the combined organic layers were washed with $1 \% \mathrm{HCl}(15 \mathrm{~mL}), \mathrm{Cu}\left(\mathrm{NO}_{3}\right)_{2}(2 \times 20 \mathrm{~mL})$ and satd. $\mathrm{NaCl}(1 \mathrm{x} 20 \mathrm{~mL})$, dried $\left(\mathrm{MgSO}_{4}\right)$, and concentrated. Purification by chromatography (8:1 hexane/ethyl acetate) afforded $55 \mathrm{mg}(100 \%)$ of 3-deoxycapsidiol acetate as a colorless oil. The purity was shown to be $>95 \%$ by ${ }^{1} \mathrm{H}$ NMR analysis. TLC $\mathrm{R}_{\mathrm{f}} 0.47$ (5:1 hexane/ethyl acetate); ${ }^{1} \mathrm{H}$ NMR (400 $\left.\mathrm{MHz}, \mathrm{CDCl}_{3}\right) \delta 6.01(\mathrm{dd}, 1 \mathrm{H}, J=6.9,2.0 \mathrm{~Hz},=\mathrm{CH}), 5.27(\mathrm{t}, 1 \mathrm{H}, J=2.9 \mathrm{~Hz}, \mathrm{CHOAc}), 4.70$ (app quintet, $\left.1 \mathrm{H}, J=1.5 \mathrm{~Hz},=\mathrm{CH}_{2}\right), 4.67$ (app septet, $\left.1 \mathrm{H}, J=1.0 \mathrm{~Hz},=\mathrm{CH}_{2}\right), 2.24(\mathrm{tt}, 1 \mathrm{H}, J=14.0,4.5 \mathrm{~Hz}, \mathrm{CH}$ or $\left.\mathrm{CH}_{2}\right), 2.05$ (tt, $\left.1 \mathrm{H}, \mathrm{J}=12.6,3.3 \mathrm{~Hz}\right), 2.10-2.00(\mathrm{~m}, 2 \mathrm{H}), 2.01\left(\mathrm{~s}, 3 \mathrm{H}, \mathrm{CH}_{3}\right), 1.88$ (ddd, 1H, J =16.7, 11.4, $1.9 \mathrm{~Hz}, \mathrm{CH}$ and $\left.\mathrm{CH}_{2}\right), 1.77(\mathrm{tt}, 1 \mathrm{H}, J=14.6,4.1 \mathrm{~Hz}), 1.72\left(\mathrm{~s}, 3 \mathrm{H},=\mathrm{CCH}_{3}\right), 1.69(\mathrm{tt}, 1 \mathrm{H}, J=10.8,2.9 \mathrm{~Hz})$, $1.60(\mathrm{~m}, 1 \mathrm{H}), 1.43(\mathrm{~s}, 1 \mathrm{H}), 1.30(\operatorname{app~t}, 1 \mathrm{H}, J=13.3 \mathrm{~Hz}, \mathrm{H} 6 \beta), 1.25$ (s, 3H, $\left.\mathrm{CH}_{3}\right), 0.97(\mathrm{~d}, 3 \mathrm{H}, J=7.2 \mathrm{~Hz}$, $\left.\mathrm{CH}_{3}\right) ;{ }^{13} \mathrm{C} \mathrm{NMR}\left(100 \mathrm{MHz}, \mathrm{CDCl}_{3}\right) \delta 170.4,149.6,137.3,130.2,108.6,76.3,44.3,40.9,40.0,38.4,31.6$, 30.3, 26.1, 25.4, 21.8, 20.9, 16.8; FTIR (neat film) 3302.4, 2091.5, 1736.6, 1643.9, 1457.7, 1370.6, 1243.0, 1209.8, 1012.7, $955.9 \mathrm{~cm}^{-1}$; HRMS (FAB) $\mathrm{m} / \mathrm{z}$ Calcd for $\mathrm{C}_{17} \mathrm{H}_{26} \mathrm{O}_{2}(\mathrm{M})^{+}: 262.1933$, found 262.1929.

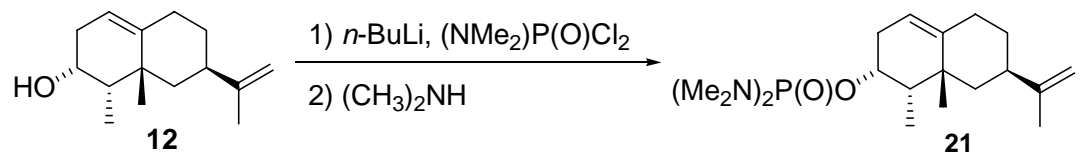

Epieremophilene 3 $\alpha$-ol Phosphoramidate (21) The procedure of Valenta et. al. was used. A solution of epieremophilene-3 $\alpha$-ol $(>85 \%, 5.3 \mathrm{mg}, 0.024 \mathrm{mmol})$, and $N, N, N^{\prime}, N^{\prime}$-tetramethylethylenediamine $(0.05$ $\mathrm{mL}, 0.039 \mathrm{~g}, 0.33 \mathrm{mmol})$ in $0.2 \mathrm{~mL}$ of DME was stirred and cooled at $0{ }^{\circ} \mathrm{C}$ as $n$-BuLi $(50 \mu \mathrm{L}, 0.070$ $\mathrm{mmol})$ in hexane was added. After $20 \mathrm{~min}, 100 \mu \mathrm{L}(0.136 \mathrm{~g}, 0.839 \mathrm{mmol})$ of dimethylphosphoramidic 
dichloride was added, and the ice bath was removed. After $2.5 \mathrm{~h}$, dimethylamine $(3 \mathrm{~mL})$ was added at -33 ${ }^{\circ} \mathrm{C}$ (acetone $/ \mathrm{H}_{2} \mathrm{O} / \mathrm{CO}_{2}$ ), and the solution was warmed to $0{ }^{\circ} \mathrm{C}$ and stirred for an additional $2 \mathrm{~h}$ at $0{ }^{\circ} \mathrm{C}$. Isolation and chromatography afforded $4.8 \mathrm{mg}(56 \%)$ of epieremophilene $3 \alpha$-ol phosphoramidate (21). The purity was estimated to be $>85 \%$ by ${ }^{1} \mathrm{H}$ NMR analysis. Data for 21 : TLC $\mathrm{R}_{\mathrm{f}} 0.20(2 \% \mathrm{MeOH} / \mathrm{ethyl}$ acetate); ${ }^{1} \mathrm{H}$ NMR $\left(500 \mathrm{MHz}, \mathrm{CDCl}_{3}\right) \delta 5.20(\mathrm{t}, 1 \mathrm{H}, J=2.5 \mathrm{~Hz},=\mathrm{CH}), 4.73(\mathrm{~m}, 1 \mathrm{H}, \mathrm{CHOP}), 4.71(\mathrm{~s}, 1 \mathrm{H}$, $=\mathrm{CH}), 4.71(\mathrm{~s}, 1 \mathrm{H},=\mathrm{CH}), 2.65\left(\mathrm{~d}, 6 \mathrm{H}, J=3.3 \mathrm{~Hz}, \mathrm{NMe}_{2}\right), 2.62\left(\mathrm{~d}, 6 \mathrm{H}, J=3.1 \mathrm{~Hz}, \mathrm{NMe}_{2}\right), 2.38(\mathrm{dt}, 1 \mathrm{H}, J$ $\left.=12.0,9.0 \mathrm{~Hz}, \mathrm{CH}_{2}\right), 2.30\left(\mathrm{dt}, 1 \mathrm{H}, J=17.5,5.5 \mathrm{~Hz}, \mathrm{CH}_{2}\right), 2.16\left(\mathrm{~m}, 1 \mathrm{H}, \mathrm{CH}_{2}\right), 2.03(\mathrm{ddd}, 1 \mathrm{H}, J=14.3$, 7.5, $\left.4.0 \mathrm{~Hz}, \mathrm{CH}_{2}\right), 1.78\left(\mathrm{~m}, 2 \mathrm{H}, \mathrm{CH}\right.$ or $\left.\mathrm{CH}_{2}\right), 1.72\left(\mathrm{~s}, 3 \mathrm{H}, \mathrm{CH}_{3}\right), 1.67$ (m, 3H, $\mathrm{CH}$ or $\left.\mathrm{CH}_{2}\right), 1.39(\mathrm{dd}, 1 \mathrm{H}, J$ $=13.8,9.7 \mathrm{~Hz}, \mathrm{H} 6 \beta), 1.21\left(\mathrm{~s}, 3 \mathrm{H}, \mathrm{CH}_{3}\right), 0.97\left(\mathrm{~d}, 3 \mathrm{H}, J=7.5 \mathrm{~Hz}, \mathrm{CH}_{3}\right)$.

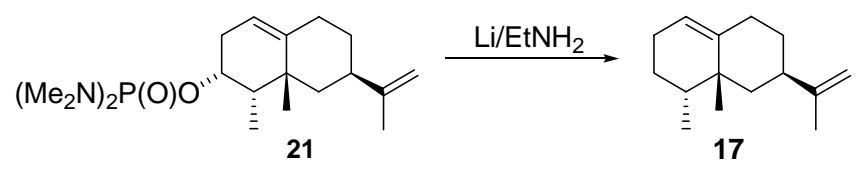

Epieremophilene (17) (Alternative to procedure presented in the Exp. Section) The procedure follows the one on page $\mathrm{S} 5$ except that the bath temperature changed to $-45^{\circ} \mathrm{C}$. Lithium pieces $(1.1 \mathrm{mg}$, $0.159 \mathrm{mmol}$ ) in $4 \mathrm{~mL}$ of dry $\mathrm{EtNH}_{2}$ were stirred and cooled at $-78{ }^{\circ} \mathrm{C}$ as epieremophilene- $3 \alpha-\mathrm{ol}$ phosphoramidate $(2.4 \mathrm{mg}, 0.00678 \mathrm{mmol})$ in $0.5 \mathrm{~mL}$ of THF was added. After $20 \mathrm{~min}, 10$ drops of $t$ butyl alcohol in $1 \mathrm{~mL}$ of THF, several drops of 3-hexyne, and satd $\mathrm{NH}_{4} \mathrm{Cl}$ were added. $\mathrm{H}_{2} \mathrm{O}(4 \mathrm{~mL})$ was added, and the product was extracted into pentane $(4 \mathrm{~mL})$. Chromatography on silica gel afforded $0.5 \mathrm{mg}$ (37\%) of epieremophilene (17) as determined by GC analysis with a cedrene standard. The GC/MS fragmentation pattern of $\mathbf{1 7}$ was identical to that of the epieremophilene prepared in experimental section.

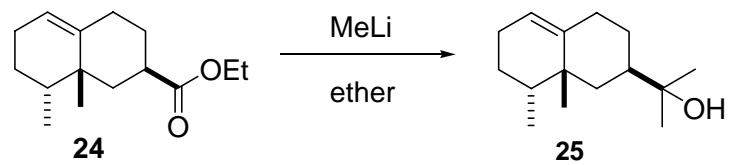

( \pm )-Epieremoligenol (25) A procedure similar to the method used by Coates and Shaw for $( \pm)$ eremoligenol was followed. ${ }^{8}$ A solution of $82.8 \mathrm{mg}(0.313 \mathrm{mmol})$ of $( \pm)$-ester $24^{9}$ in $1 \mathrm{~mL}$ of ether was stirred at $\mathrm{rt}$ as MeLi $(1 \mathrm{M}, 3 \mathrm{~mL}, 3.0 \mathrm{mmol})$ in ether was added. After $1 \mathrm{~h}$, the solution was cooled to $0{ }^{\circ} \mathrm{C}$, and $4 \mathrm{~mL}$ of satd. $\mathrm{NH}_{4} \mathrm{Cl}$ was slowly added. The aqueous layer was extracted with ether ( 2 x $\left.3 \mathrm{~mL}\right)$, and the combined organic layers were washed with $\mathrm{H}_{2} \mathrm{O}(4 \mathrm{~mL})$, dried $\left(\mathrm{Na}_{2} \mathrm{SO}_{4}\right)$ and concentrated at reduced pressure. Chromatography on silica gel (6:1 hexane/ethyl acetate) provided $46.6 \mathrm{mg}$ (65\%) of 8 Coates, R. M.; Shaw, J. E. J. Org. Chem. 1970, 35, 2597.

9 Ester 24 was obtained from the UI sample collection. The preparative procedure for this compound is given in reference 7 . 
epieremoligenol as a colorless oil. ${ }^{1} \mathrm{H}$ NMR and GC analyses determined that the epieremoligenol was $85 \%$ pure with residual hexane peaks $(\sim 15 \%)$. ${ }^{1} \mathrm{H} \mathrm{NMR}\left(500 \mathrm{MHz}, \mathrm{CDCl}_{3}\right) \delta 5.35$ (app sextet, $1 \mathrm{H}, J=2.3$ $\mathrm{Hz},=\mathrm{CH}), 2.37\left(\mathrm{~m}, 1 \mathrm{H}, \mathrm{CH}_{2}\right), 2.02\left(\mathrm{~m}, 2 \mathrm{H}, \mathrm{CH}_{2}\right), 1.89(\mathrm{dtt}, 1 \mathrm{H}, J=17.5,5.7,3.2 \mathrm{~Hz}), 1.73\left(\mathrm{~m}, 2 \mathrm{H}, \mathrm{CH}_{2}\right)$, 1.69 (dq, $1 \mathrm{H}, J=14.8,2.0 \mathrm{~Hz}, \mathrm{CH}_{2}$ ), 1.55 (quintet of d, 1H, $J=7.0,3.5 \mathrm{~Hz}, \mathrm{CH}$ ), 1.46 (tt, $1 \mathrm{H}, J=12.5$, $\left.4.0 \mathrm{~Hz}, \mathrm{CH}_{2}\right), 1.40\left(\mathrm{dq}, 1 \mathrm{H}, J=12.3,2.3 \mathrm{~Hz}, \mathrm{CH}_{2}\right), 1.35$ (bs, $\left.1 \mathrm{H}, \mathrm{OH}\right), 1.28$ (ddt, 1H, J=6.3, 6.2, $6.0 \mathrm{~Hz}$, $\mathrm{CH}), 1.15$ (s, 3H, $\left.\mathrm{CH}_{3}\right), 1.14\left(\mathrm{~s}, 3 \mathrm{H}, \mathrm{CH}_{3}\right), 1.14\left(\mathrm{~s}, 3 \mathrm{H}, \mathrm{CH}_{3}\right), 1.05$ (app t, 1H, J = $\left.12.7 \mathrm{~Hz}, \mathrm{H} 6 \beta\right), 0.95$ (d, $\left.3 \mathrm{H}, J=7.0 \mathrm{~Hz}, \mathrm{CH}_{3}\right), 0.88\left(\mathrm{t}, 6 \mathrm{H}\right.$, hexane $\left.\mathrm{CH}_{3}\right) ;{ }^{13} \mathrm{C} \mathrm{NMR}\left(125 \mathrm{MHz}, \mathrm{CDCl}_{3}\right) \delta 141.2,119.9,73.1,43.5$, $39.7,36.7,36.0,31.6,29.6,28.4,27.0,26.7,24.3,22.8,15.5$.

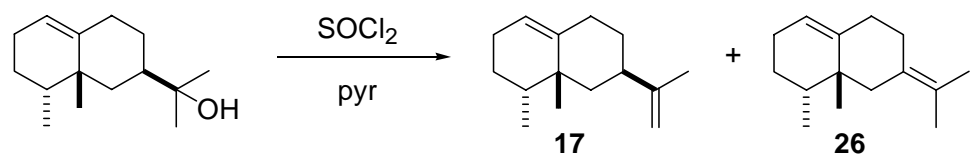

( \pm )-Epieremophilene (17) A procedure similar to that used by Coates and Shaw for $( \pm)$-eremophilene was followed. A solution of $12.9 \mathrm{mg}(0.0580 \mathrm{mmol})$ of epieremoligenol in $0.5 \mathrm{~mL}$ of pyridine was stirred and cooled at $0{ }^{\circ} \mathrm{C}$ as thionyl chloride $(12 \mu \mathrm{L}, 20 \mathrm{mg}, 0.168 \mathrm{mmol})$ was added. After 20 min the solution was poured into $1 \mathrm{~mL}$ of $\mathrm{H}_{2} \mathrm{O}$, the aqueous layer was extracted with pentane $(3 \mathrm{x} 2 \mathrm{~mL})$, and the combined organic layers were washed with $1 \% \mathrm{HCl}(6 \mathrm{~mL})$ and satd $\mathrm{NaHCO}_{3}(6 \mathrm{~mL})$, and dried $\left(\mathrm{Na}_{2} \mathrm{SO}_{4}\right)$. GC analysis showed that the crude extracts contained a 2.7:1 mixture of racemic epieremophilene (17) and the isopropylidene isomer (26). Purification by chromatography on silica gel (pentane) and GC analysis with cedrene as standard gave $10.1 \mathrm{mg}(87 \%)$ of a 2.8:1 mixture of epieremophilene and the other 26. The olefins were separated by chromatography on $15 \% \mathrm{AgNO}_{3}$-silica gel with 50:1 pentane/ether. ${ }^{1} \mathrm{H}$ NMR and GC analyses determined the purity of $\mathbf{1 7}$ to be $95 \%$. Data for 17: TLC $\mathrm{R}_{\mathrm{f}} 0.43\left(\mathrm{AgNO}_{3}\right.$-silica gel, 50:1 pentane/ether); ${ }^{1} \mathrm{H}$ NMR $\left(500 \mathrm{MHz}, \mathrm{C}_{6} \mathrm{D}_{6}\right) \delta 5.40(\operatorname{td}, 1 \mathrm{H}, J=$ 3.7, $1.5 \mathrm{~Hz},=\mathrm{CH}), 4.86(\mathrm{~s}, 1 \mathrm{H},=\mathrm{CH}), 4.82(\mathrm{~s}, 1 \mathrm{H},=\mathrm{CH}), 2.47(\mathrm{~m}, 1 \mathrm{H}, \mathrm{CH}), 2.14(\mathrm{tt}, 1 \mathrm{H}, J=8.7,5.5 \mathrm{~Hz}$, $\left.\mathrm{CH}_{2}\right), 1.98\left(\mathrm{~m}, 2 \mathrm{H}, \mathrm{CH}_{2}\right), 1.89$ (dtt, 1H, J=17.5, 6.0, $\left.3.5 \mathrm{~Hz}, \mathrm{CH}_{2}\right), 1.72(\mathrm{~m}, 1 \mathrm{H}, \mathrm{CH}), 1.68\left(\mathrm{~s}, 3 \mathrm{H}, \mathrm{CH}_{3}\right)$, 1.66 1.58 (m, 4H, $\mathrm{CH}_{2}$ and $\left.\mathrm{CH}\right), 1.48 \sim 1.40\left(\mathrm{~m}, 2 \mathrm{H}, \mathrm{CH}_{2}\right), 1.15\left(\mathrm{~s}, 3 \mathrm{H}, \mathrm{CH}_{3}\right), 0.92(\mathrm{~d}, 3 \mathrm{H}, J=7.0 \mathrm{~Hz}$, $\mathrm{CH}_{3}$ ); The GC/MS fragmentation pattern of $( \pm)-\mathbf{1 7}$ was identical to that for the epieremophilene prepared by reduction of the tosyl hydrazone and phosphoramidate.

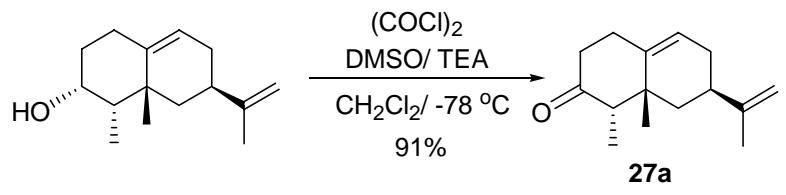


Epiaristolochene-3-one (27a) Method A The general procedure of Heathcock was followed. ${ }^{10}$ Oxalyl chloride (47 mg, $32 \mu \mathrm{L}, 0.37 \mathrm{mmol})$ and DMSO (55 mg, $50 \mu \mathrm{L}, 0.71 \mathrm{mmol})$ were dissolved in $\mathrm{CH}_{2} \mathrm{Cl}_{2}(3$ $\mathrm{mL}$ ), and the solution was cooled to $-78{ }^{\circ} \mathrm{C}$. 1-Deoxycapsidiol (62 mg, $\left.0.28 \mathrm{mmol}\right)$ in $\mathrm{CH}_{2} \mathrm{Cl}_{2}(6 \mathrm{~mL})$ was then added slowly. The reaction mixture was stirred at $-78{ }^{\circ} \mathrm{C}$ for $20 \mathrm{~min}$, triethylamine $(145 \mathrm{mg}, 200$ $\mu \mathrm{L}, 1.44 \mathrm{mmol}$ ) was added, and the solution was warmed up to $\mathrm{rt}$ for another $20 \mathrm{~min}$. The solution was washed with brine $(10 \mathrm{~mL})$, and the aqueous layer was extracted with $\mathrm{Et}_{2} \mathrm{O}(3 \mathrm{x} 20 \mathrm{~mL})$. The combined organic layers was dried $\left(\mathrm{MgSO}_{4}\right)$ and concentrated. Purification of the crude product by flash chromatography (15:1 hexane/ethyl acetate) provided ketone $27 \mathbf{a}(46 \mathrm{mg}, 91 \%)$ as a clear oil and recovered starting material $(8 \mathrm{mg})$. The purity of the product was shown to be $95 \%$ by ${ }^{1} \mathrm{H}$ NMR analysis. Data for ketone 27a: TLC R 0.19 (15:1 hexane/ethyl acetate); GC (99\%, Program 1); ${ }^{1} \mathrm{H}$ NMR (400 $\left.\mathrm{MHz}, \mathrm{CDCl}_{3}\right) \delta 5.79\left(\mathrm{dd}, 1 \mathrm{H}, J=6.5,1.8 \mathrm{~Hz},=\mathrm{CH}\right.$ ), 4.72 (quintet, $1 \mathrm{H}, J=1.4 \mathrm{~Hz},=\mathrm{CH}_{2}$ ), 4.69 (septet, $\left.1 \mathrm{H}, J=0.9 \mathrm{~Hz},=\mathrm{CH}_{2}\right), 2.55(\mathrm{~m}, 1 \mathrm{H}), 2.50(\mathrm{dd}, 1 \mathrm{H}, J=12.7,5.7 \mathrm{~Hz}, \mathrm{H} 4 \alpha), 2.34(\mathrm{~m}, 1 \mathrm{H}), 2.32(\mathrm{tt}, 1 \mathrm{H}, J=$ 14.9, $3.7 \mathrm{~Hz}), 2.25(\mathrm{~m}, 1 \mathrm{H}), 2.18(\mathrm{dd}, J=7.3,1.5 \mathrm{~Hz}), 2.14(\mathrm{~m}, 1 \mathrm{H}), 1.87(\mathrm{tdd}, 1 \mathrm{H}, J=11.2,3.8,1.6 \mathrm{~Hz})$, $1.73\left(\mathrm{~s}, 3 \mathrm{H}, \mathrm{CH}_{3}\right), 1.69$ (ddd, $\left.1 \mathrm{H}, \mathrm{J}=14.1,3.2,2.5 \mathrm{~Hz}\right), 1.37$ (app t, $\left.1 \mathrm{H}, J=13.7 \mathrm{~Hz}, \mathrm{H} 6 \beta\right), 1.15$ (d, 3H, $J$ $\left.=7.2 \mathrm{~Hz}, \mathrm{CH}_{3}\right), 1.03\left(\mathrm{~s}, 3 \mathrm{H}, \mathrm{CH}_{3}\right) ;{ }^{13} \mathrm{C} \mathrm{NMR}\left(100 \mathrm{MHz}, \mathrm{CDCl}_{3}\right) \delta 149.2,136.3,124.3,108.9,108.8$, $58.6,42.1,39.7,39.4,37.7,30.3,30.2,30.0,20.9,16.2$.

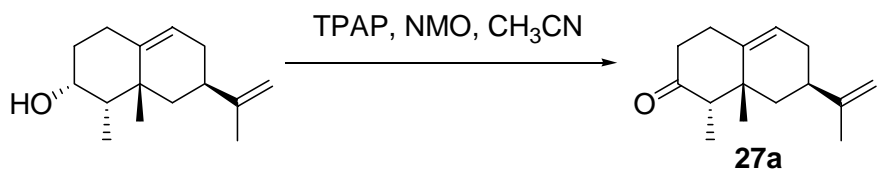

Epiaristolochene 3-one (27a) Method B. A suspension of 4 A molecular sieves (5 mg) in a solution of 1deoxycapsidiol (10 mg, $0.045 \mathrm{mmol})$, NMO (18 mg, $0.153 \mathrm{mmol})$, and $\mathrm{CH}_{3} \mathrm{CN}(0.5 \mathrm{~mL})$ was stirred at $\mathrm{rt}$ as TPAP (1.6 mg, $0.0045 \mathrm{mmol})$ in $0.5 \mathrm{~mL}$ of $\mathrm{CH}_{3} \mathrm{CN}$ was added. ${ }^{11}$ After $1 \mathrm{~h}, \mathrm{H}_{2} \mathrm{O}(5 \mathrm{~mL})$ and ether (5 $\mathrm{mL})$ were added, and the aqueous layer was extracted with ether $(2 \mathrm{x}, 5 \mathrm{~mL})$. The combined organic layers were washed with $\mathrm{H}_{2} \mathrm{O}(15 \mathrm{~mL})$, dried $\left(\mathrm{MgSO}_{4}\right)$, and concentrated. Purification by chromatography on silica gel (2:1 hexane/ethyl acetate) gave $4.4 \mathrm{mg}$ (44\%) of ketone 27a. The NMR data of $\mathbf{2 7} \mathbf{a}$ are consistent with those described before.

10 Takai, K.; Heathcock, C. H. J. Org. Chem. 1985, 50, 3247.

11 Cherif, A.; Farquhar, D. J. Med. Chem. 1992, 35, 3208. 


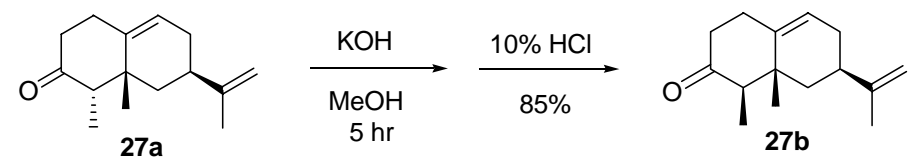

Eremophila-9(10), 11(12)-diene-3-one (27b) A solution of ketone 27a (20 mg, $0.092 \mathrm{mmol})$ and $\mathrm{KOH}$ (30 mg, $0.54 \mathrm{mmol})$ in $\mathrm{MeOH}(1.5 \mathrm{~mL})$ was stirred at $\mathrm{rt}$ for $2 \mathrm{~h} . \mathrm{Et}_{2} \mathrm{O}(5 \mathrm{~mL})$ and $10 \% \mathrm{HCl}(5 \mathrm{~mL})$ were added, and the mixture was stirred for $20 \mathrm{~min}$. The aqueous layer was extracted with $\mathrm{Et}_{2} \mathrm{O}(3 \mathrm{x} 30 \mathrm{~mL})$. The ether layers were combined, washed with brine $(10 \mathrm{~mL})$, dried $\left(\mathrm{MgSO}_{4}\right)$, and evaporated to give a 11:1 (NMR cis:trans) mixture of ketones. Purification by flash chromatography (20:1 hexane: ethyl acetate) gave the cis isomer (11 mg, 75\%, containing $~ 5 \%$ grease) and trans isomer (5 $\mathrm{mg}, 7 \%)$ as colorless oils. Data for cis isomer 27b: TLC R 0.49 (6:1 hexane/ethyl acetate); GC (100\%, Program 1); ${ }^{1} \mathrm{H}$ NMR $\left(500 \mathrm{MHz}_{\mathrm{CDCl}}\right) \delta 5.59(\mathrm{dt}, 1 \mathrm{H}, J=6.0,1.7 \mathrm{~Hz},=\mathrm{CH}), 4.75\left(\mathrm{t}, 1 \mathrm{H}, J=1.3 \mathrm{~Hz},=\mathrm{CH}_{2}\right), 4.73$ $\left(\mathrm{s}, 1 \mathrm{H},=\mathrm{CH}_{2}\right), 3.76(\mathrm{~m}$, grease $\mathrm{H}), 2.66\left(\operatorname{app~q}, 1 \mathrm{H}, J=6.8 \mathrm{~Hz}, \mathrm{CH}_{2}\right), 2.57\left(\mathrm{~m}, 1 \mathrm{H}, \mathrm{CH}_{2}\right), 2.56(\mathrm{~m}, 1 \mathrm{H})$, $2.34(\operatorname{app~q}, 1 \mathrm{H}, J=10.9 \mathrm{~Hz}, \mathrm{CH}), 2.33(\mathrm{tt}, 1 \mathrm{H}, J=14.5,7.5 \mathrm{~Hz}), 2.17$ (m, 2H), 1.97(dddd, 1H, $J=16.8$, 11.3, 3.7, 1.9 Hz), 1.93 (dt, 1H, $J=13.9,2.1 \mathrm{~Hz}), 1.75$ (s, 3H, $\left.\mathrm{CH}_{3}\right), 1.27$ (app t, 1H, $\left.J=12.8 \mathrm{~Hz}, \mathrm{H} 6 \beta\right)$, $0.96\left(\mathrm{~d}, 3 \mathrm{H}, J=6.9 \mathrm{~Hz}, \mathrm{CH}_{3}\right.$ ), 0.86 (s, 3H, $\mathrm{CH}_{3}$ ); FTIR (neat film), 2964.2, 2880.2, 1709.4, 1632.9, 1447.9,1258.2, 1177.6, 887.1 $\mathrm{cm}^{-1}$; HRMS (EI) m/z Calcd for $\mathrm{C}_{15} \mathrm{H}_{22} \mathrm{O} \mathrm{M}^{+}$: 218.1671, found 218.1677.

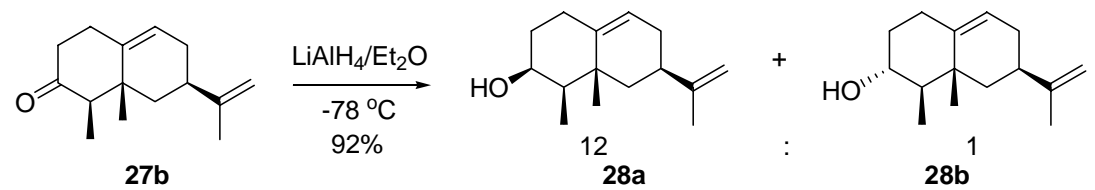

Eremophila-9(10), 11(12)-diene 3 $\beta$-ol (28a) and eremophila-9(10), 11(12)-dien-3 $\alpha-o l$ (28b). A solution of ketone $\mathbf{2 7 b}(27 \mathrm{mg}, 0.12 \mathrm{mmol})$ in $1.5 \mathrm{~mL}$ of $\mathrm{Et}_{2} \mathrm{O}$ was stirred and cooled at $-78{ }^{\circ} \mathrm{C}$ as $\mathrm{LiAlH}_{4}$ (6 $\mathrm{mg}, 0.15 \mathrm{mmol}$ ) was added. After $40 \mathrm{~min}$, the reaction mixture was poured onto ice $(25 \mathrm{~g}), \mathrm{Et}_{2} \mathrm{O}$ was added, and the $\mathrm{pH}$ was adjusted to around 7 by adding $10 \% \mathrm{HCl}(2 \mathrm{~mL})$. The aqueous layer was extracted with ether $(3 \times 20 \mathrm{~mL})$. The combined organic layers were washed with brine $(15 \mathrm{~mL})$, dried $\left(\mathrm{MgSO}_{4}\right)$, and concentrated affording $28 \mathrm{mg}$ of crude alcohols. Purification by flash chromatography on silica gel (20:1 hexane/ethyl acetate) provided $22 \mathrm{mg}$ of cis isomer and $2 \mathrm{mg}$ of trans isomer: total yield $24 \mathrm{mg}$ (92\%). Data for cis isomer 28a: TLC $\mathrm{R}_{\mathrm{f}} 0.31\left(6: 1\right.$ hexane/ethyl acetate); $[\alpha]^{25}{ }_{\mathrm{D}}=+3.32\left(\mathrm{c}=1.00, \mathrm{CHCl}_{3}\right)$; ${ }^{1} \mathrm{H}$ NMR $\left(400 \mathrm{MHz}, \mathrm{CDCl}_{3}\right) \delta 5.42(\mathrm{dt}, 1 \mathrm{H}, J=6.1,1.7 \mathrm{~Hz},=\mathrm{CH}), 4.71$ (quintet, $\left.1 \mathrm{H}, J=1.6 \mathrm{~Hz},=\mathrm{CH}_{2}\right)$, 4.69 (septet, $\left.1 \mathrm{H}, J=1.0 \mathrm{~Hz},=\mathrm{CH}_{2}\right), 3.92$ (q, $\left.1 \mathrm{H}, J=3.0 \mathrm{~Hz}, \mathrm{CHOH}\right), 2.59$ (tt, $\left.1 \mathrm{H}, J=13.2,3.3 \mathrm{~Hz}, \mathrm{H} 2 \beta\right)$, $2.04(\mathrm{t}, 1 \mathrm{H}, J=1.0 \mathrm{~Hz}), 2.02-1.84(\mathrm{~m}, 5 \mathrm{H}), 1.71(\mathrm{td}, 1 \mathrm{H}, J=7.1,3.4 \mathrm{~Hz}, \mathrm{CH}), 1.73\left(\mathrm{~s}, 3 \mathrm{H}, \mathrm{CH}_{3}\right), 1.56$ (tdd, $1 \mathrm{H}, J=13.9,4.7,3.3 \mathrm{~Hz}), 1.42(\mathrm{~s}, 1 \mathrm{H}, \mathrm{CHOH}), 1.17\left(\mathrm{~s}, 3 \mathrm{H}, \mathrm{CH}_{3}\right), 1.03(\mathrm{dd}, 1 \mathrm{H}, J=13.4,12.6 \mathrm{~Hz}$, 
$\mathrm{H} 6 \beta), 1.01\left(\mathrm{~d}, 3 \mathrm{H}, J=7.2 \mathrm{~Hz}, \mathrm{CH}_{3}\right) ;{ }^{13} \mathrm{C} \mathrm{NMR}\left(126 \mathrm{MHz}, \mathrm{CDCl}_{3}\right) \delta 150.0,146.0,118.4,108.4,72.7$, 42.4, 40.9, 38.3, 36.9, 31.4, 28.1, 27.2, 24.2, 21.1, 11.9; FTIR (neat film), 3400.7, 2936.9, 2098.5, 1643.9, 1450.2, 1371.9, 1169.5, 1018.3, 994.4, 957.0, 929.9, $887.0 \mathrm{~cm}^{-1}$; HRMS (EI) m/z Calcad for $\mathrm{C}_{15} \mathrm{H}_{22} \mathrm{O} \mathrm{M}^{+}$: 220.1827, found 220.1821. Data for trans isomer 28b: TLC $\mathrm{R}_{\mathrm{f}} 0.21$ (6:1 hexane/ethyl acetate); ${ }^{1} \mathrm{H}$ NMR $\left(400 \mathrm{MHz}, \mathrm{CDCl}_{3}\right) \delta 5.41(\mathrm{dt}, 1 \mathrm{H}, J=6.2,1.6 \mathrm{~Hz},=\mathrm{CH}), 4.71\left(\mathrm{~m}, 1 \mathrm{H},=\mathrm{CH}_{2}\right), 4.69\left(\mathrm{~m}, 1 \mathrm{H},=\mathrm{CH}_{2}\right), 3.58$ $(\mathrm{td}, 1 \mathrm{H}, J=10.6,4.7 \mathrm{~Hz}, \mathrm{CHOH}), 2.28\left(\mathrm{tt}, 1 \mathrm{H}, J=13.1,3.8 \mathrm{~Hz}, \mathrm{CH}_{2}\right), 2.16-1.97$ (m, 4H), 1.96 (dt, $1 \mathrm{H}, J$ $=13.5,2.2 \mathrm{~Hz}, \mathrm{CH}), 1.87(\mathrm{dddd}, 1 \mathrm{H}, J=16.5,11.1,3.1,1.9 \mathrm{~Hz}), 1.73\left(\mathrm{~s}, 3 \mathrm{H}, \mathrm{CH}_{3}\right), 1.56(\mathrm{~s}, 1 \mathrm{H}, \mathrm{CHOH})$, $1.48(\mathrm{dq}, 1 \mathrm{H}, J=9.8,6.4 \mathrm{~Hz}), 1.27(\mathrm{~m}, 1 \mathrm{H}), 1.10(\mathrm{dd}, 1 \mathrm{H}, J=13.5,12.7 \mathrm{~Hz}, \mathrm{H} 6 \beta), 1.00(\mathrm{~d}, 3 \mathrm{H}, J=6.6$ $\left.\mathrm{Hz}, \mathrm{CH}_{3}\right), 0.93\left(\mathrm{~s}, 3 \mathrm{H}, \mathrm{CH}_{3}\right)$.

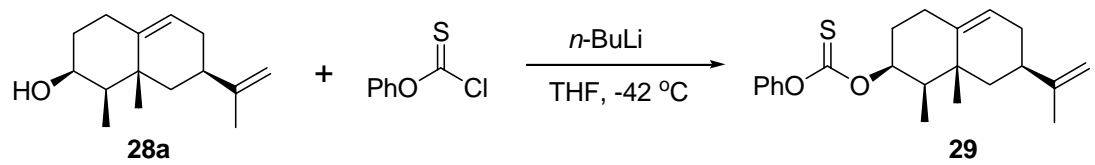

Eremophila-9(10), 11(12)-dien-3 $\beta-0 l$ Phenylthionocarbonate (29) A solution of eremophila-9(10), 11(12)-diene-3 $\beta$-ol (25 mg, $0.11 \mathrm{mmol})$ in THF (3.0 mL) was stirred and cooled at $-42{ }^{\circ} \mathrm{C}$ as $n$-BuLi (100 $\mu \mathrm{L}, 1.6 \mathrm{M}$ in hexane, $0.16 \mathrm{mmol})$ was added. After $20 \mathrm{~min}$ at $-42{ }^{\circ} \mathrm{C}$, phenyl chlorothionoformate $(50 \mu \mathrm{L}$, $0.33 \mathrm{mmol}$ ) was added slowly over $2 \mathrm{~min}$. The reaction mixture was warmed up to $\mathrm{rt}$ and stirred for 30 min. The solution was poured onto a mixture of ice water and ether $(15 \mathrm{~mL} / 20 \mathrm{~mL})$, and the aqueous layer was extracted with ether $(3 \times 30 \mathrm{~mL})$. The combined organic layers were washed with satd. $\mathrm{NaCl}$ $(20 \mathrm{~mL})$ and dried $\left(\mathrm{MgSO}_{4}\right)$. Purification by flash chromatography (50:1 hexane/ethyl acetate) afforded $35 \mathrm{mg}$ (83\%, containing $\sim 5 \%$ hexane) of PTC derivative (29) as a yellow oil: TLC $\mathrm{R}_{\mathrm{f}}$ 0.18 (50:1 hexane/ethyl acetate); ${ }^{1} \mathrm{H} \mathrm{NMR}\left(\mathrm{CDCl}_{3}, 400 \mathrm{MHz}\right) \delta 7.47 \sim 7.40(\mathrm{~m}, 2 \mathrm{H}$, phenyl $\mathrm{H}), 7.32 \sim 7.27(\mathrm{~m}, 1 \mathrm{H}$, phenyl H), 7.17 7.12 (m, 2H, phenyl H), 5.59 (app q, 1H, J=3.0 Hz, CHO), 5.49 (dt, $1 \mathrm{H}, J=6.0,1.2 \mathrm{~Hz}$, $=\mathrm{CH}$ ), 4.74 (quintet, $\left.1 \mathrm{H}, J=1.6 \mathrm{~Hz},=\mathrm{CH}_{2}\right), 4.72\left(\right.$ app sextet, $\left.1 \mathrm{H}, J=0.9 \mathrm{~Hz},=\mathrm{CH}_{2}\right), 2.43(\mathrm{tt}, 1 \mathrm{H}, J=$

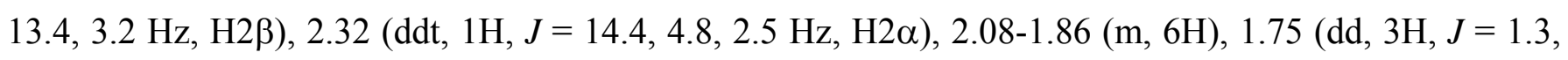
$\left.0.8 \mathrm{~Hz}, \mathrm{CH}_{3}\right), 1.61$ (tdd, $\left.1 \mathrm{H}, J=14.1,4.6,3.2 \mathrm{~Hz}\right), 1.10$ (dd, 1H, J=13.6, $\left.12.6 \mathrm{~Hz}, \mathrm{H} 6 \beta\right), 1.00$ (d, 3H, $J=$ $\left.7.0 \mathrm{~Hz}, \mathrm{CH}_{3}\right), 0.95\left(\mathrm{~s}, 3 \mathrm{H}, \mathrm{CH}_{3}\right), 0.88\left(\mathrm{t}\right.$, hexane $\left.\mathrm{CH}_{3}\right) ;{ }^{13} \mathrm{C} \mathrm{NMR} \delta$ 194.6, 153.3, 149.6, 144.6, 129.5, 126.4, 121.9, 119.5, 108.7, 87.0, 42.0, 40.4, 39.1, 38.2, 32.9, 31.3, 27.5, 23.3, 21.1, 11.4; FTIR (neat film) 3070, 2873, 1639, 1586, 1488, 1366, 1268, 1191, 1002, 886, 766, $689 \mathrm{~cm}^{-1}$; HRMS (EI) m/z Calcd for $\mathrm{C}_{22} \mathrm{H}_{28} \mathrm{O}_{2} \mathrm{~S}: 356.1810$, found 356.1813 . 


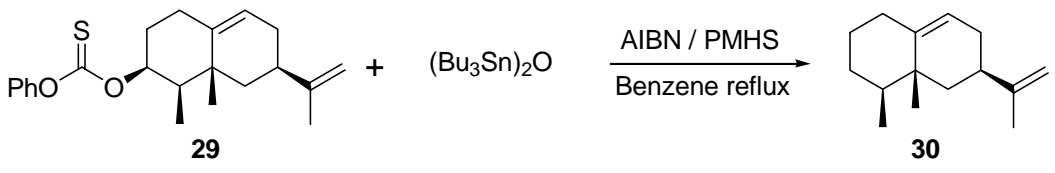

(-)-Eremophila-9(10), 11(12)-diene (30) The general procedure of Fu was followed. ${ }^{12}$ To a solution of (29) $(18 \mathrm{mg}, 0.05 \mathrm{mmol})$ in $2 \mathrm{~mL}$ of benzene at $\mathrm{rt}$ were added a benzene solution $(4 \mathrm{~mL})$ of $\left(\mathrm{Bu}_{3} \mathrm{Sn}\right)_{2} \mathrm{O}(10$ $\mu \mathrm{L}, 0.02 \mathrm{mmol}$ ), AIBN (2,2'-azobisisobutyronitrile, $3 \mathrm{mg}, 0.01 \mathrm{mmol}$ ), PMHS (polymethylhydrosiloxane) (200 mg, $3.34 \mathrm{mmol}$ ) and 1-butanol ( $32 \mathrm{mg}, 40 \mu \mathrm{L}, 0.44 \mathrm{mmol}$ ). After stirring for $5 \mathrm{~min}$, the reaction mixture was heated to reflux. After $5 \mathrm{~h}$, the solution was cooled to $\mathrm{rt}$ and concentrated. THF $(4 \mathrm{~mL})$ and aq. $\mathrm{NaOH}(2 \mathrm{M}, 3 \mathrm{~mL})$ were added. The mixture was stirred for another $3 \mathrm{~h}$ at $\mathrm{rt}$, and the aqueous layer was extracted with pentane $(3 \times 15 \mathrm{~mL})$. The combined organic extracts were concentrated to ca. $3 \mathrm{~mL}$. Purification by flash chromatography on silica gel (pentane) afforded (-)-eremophila-9(10), 11(12)-diene ( $85 \%$ pure determined by GC). Further purification by $15 \% \mathrm{AgNO}_{3}$-silica gel ( $15 \mathrm{~mL}$ of pentane followed by $15 \mathrm{~mL}$ of ether) gave the previously unknown (-)-eremophila-9(10), 11(12)-diene (30, $7.7 \mathrm{mg}, 75 \%$, $97 \%$ pure determined by GC): TLC $\mathrm{R}_{\mathrm{f}} 0.70$ (pentane); $[\alpha]^{25}{ }_{\mathrm{D}}=-11.8\left(\mathrm{c}=0.30, \mathrm{CHCl}_{3}\right) ;{ }^{1} \mathrm{H}$ NMR $(500$ $\left.\mathrm{MHz}, \mathrm{CDCl}_{3}\right) \delta 5.35(\mathrm{tt}, 1 \mathrm{H}, J=6.3,1.8 \mathrm{~Hz},=\mathrm{CH}), 4.70\left(\mathrm{~m}, 2 \mathrm{H},=\mathrm{CH}_{2}\right), 2.24-2.11\left(\mathrm{~m}, 2 \mathrm{H}, \mathrm{CH}_{2}\right), 1.99$ (m, 1H), $1.93(\mathrm{t}, 1 \mathrm{H}, J=2.4 \mathrm{~Hz}), 1.91(\mathrm{t}, 1 \mathrm{H}, J=2.3 \mathrm{~Hz}), 1.87$ (tdd, 1H, $J=11.3,3.3,2.0 \mathrm{~Hz}), 1.78$ (d of septet, $1 \mathrm{H}, J=12.3,2.3 \mathrm{~Hz}), 1.74\left(\mathrm{~m}, 3 \mathrm{H}, \mathrm{CH}_{3}\right), 1.66(\mathrm{~m}, 1 \mathrm{H}), 1.51(\mathrm{~m}, 1 \mathrm{H}), 1.41(\mathrm{qd}, 1 \mathrm{H}, J=11.8,4.0$ $\mathrm{Hz}), 1.27$ (qt, $1 \mathrm{H}, J=12.5,4.5 \mathrm{~Hz}), 1.12(\operatorname{app} \mathrm{t}, 1 \mathrm{H}, J=13.2 \mathrm{~Hz}, \mathrm{H} 6 \beta), 0.93\left(\mathrm{~s}, 3 \mathrm{H}, \mathrm{CH}_{3}\right), 0.80(\mathrm{~d}, 3 \mathrm{H}, J$ $\left.=6.6 \mathrm{~Hz}, \mathrm{CH}_{3}\right) ;{ }^{13} \mathrm{C} \mathrm{NMR}\left(\mathrm{CDCl}_{3}, 500 \mathrm{MHz}\right) \delta 167.0,150.4,146.4,117.9,108.3,39.6,38.7,37.7,32.3$, 31.5, 31.4, 31.3, 29.2, 21.1, 20.8, 15.7. HRMS (EI) m/z Calcd for $\mathrm{C}_{15} \mathrm{H}_{24} \mathrm{M}^{+}: 204.1878$, found 204.1876.

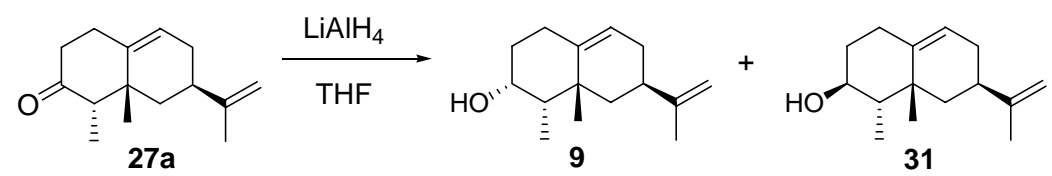

Epiaristolochene-3 $\beta$-ol (31) A solution of $4.4 \mathrm{mg}(0.020 \mathrm{mmol})$ of ketone $27 \mathrm{a}$ in $1 \mathrm{~mL}$ of THF was stirred at $0{ }^{\circ} \mathrm{C}$ as $\mathrm{LiAlH}_{4}(14 \mathrm{mg}, 0.10 \mathrm{mmol})$ was added. After $0.5 \mathrm{~h}, 14 \mu \mathrm{L}$ of $\mathrm{H}_{2} \mathrm{O}, 14 \mu \mathrm{L}$ of $15 \%$ $\mathrm{NaOH}$, and $42 \mu \mathrm{L}$ of $\mathrm{H}_{2} \mathrm{O}$ were added, ${ }^{13}$ and the precipitate was filtered and washed with ether (3x 10 $\mathrm{mL})$. Purification by chromatography on silica gel (4:1 hexane/ethyl acetate) afforded $3.3 \mathrm{mg}$ ( $70 \%)$ of 31 and $1.0 \mathrm{mg}$ (23\%) of deoxycapsidiol (9). The purity of alcohol 31 was shown to be $95 \%$ by ${ }^{1} \mathrm{H}$ NMR

12 Tormo, J.; Fu, G. C. Org. Synth. 2002, 78, 239-248.

13 Coates, R. M.; Ley, D. A.; Cavender, P. L. J. Org. Chem. 1978, 43, 4915. 
(containing $\sim 5 \%$ hexane) and GC analyses. Data for 31: TLC $\mathrm{R}_{\mathrm{f}} 0.28$ (4:1 hexane/ethyl acetate); GC $(13.1 \mathrm{~min}, 100 \%) ;[\alpha]_{\mathrm{D}}=+0.25\left(\mathrm{c}=0.55, \mathrm{CHCl}_{3}\right) ;{ }^{1} \mathrm{H} \mathrm{NMR}\left(500 \mathrm{MHz}, \mathrm{CDCl}_{3}\right) \delta 5.58(\mathrm{dt}, 1 \mathrm{H}, J=6.5$, 2.0, $=\mathrm{CH}), 4.69(\mathrm{~m}, 1 \mathrm{H},=\mathrm{CH}), 4.67(\mathrm{~m}, 1 \mathrm{H},=\mathrm{CH}), 3.83(\mathrm{q}, 1 \mathrm{H}, \mathrm{J}=3.0, \mathrm{CHOH}), 2.64\left(\mathrm{~m}, 1 \mathrm{H}, \mathrm{CH}_{2}\right), 2.21(\mathrm{tt}$, $\left.1 \mathrm{H}, J=12.0,4.0, \mathrm{CH}_{2}\right), 2.01\left(\mathrm{~m}, 1 \mathrm{H}, \mathrm{CH}_{2}\right), 1.87\left(\mathrm{dt}, 1 \mathrm{H}, J=16.5,4.0, \mathrm{CH}_{2}\right), 1.82\left(\mathrm{~m}, 2 \mathrm{H}, \mathrm{CH}_{2}\right), 1.73,(\mathrm{~s}$, $\left.3 \mathrm{H}, \mathrm{CH}_{3}\right), 1.70(\mathrm{~m}, 2 \mathrm{H}) .1 .63(\mathrm{q}, 1 \mathrm{H}, J=7.0, \mathrm{CH}), 1.56(\mathrm{bs}, 1 \mathrm{H}, \mathrm{OH}), 1.39\left(\mathrm{~s}, 3 \mathrm{H}, \mathrm{CH}_{3}\right), 1.20(\operatorname{app~t}, 1 \mathrm{H}, J=$ 13.5, H6 3 ), 0.95(d, 3H, $\left.J=7.5, \mathrm{CH}_{3}\right), 0.88\left(\mathrm{t}\right.$, hexane $\left.\mathrm{CH}_{3}\right) ;{ }^{13} \mathrm{C} \mathrm{NMR}\left(100 \mathrm{MHz}, \mathrm{CDCl}_{3}\right) \delta$ 74.6, 48.3, 44.9, 39.8, 38.4, 37.7, 30.4, 30.2, 26.8, 20.9, 17.3, 17.3. The ${ }^{1} \mathrm{H}$ NMR data for 9 were similar to those described on page S4. 


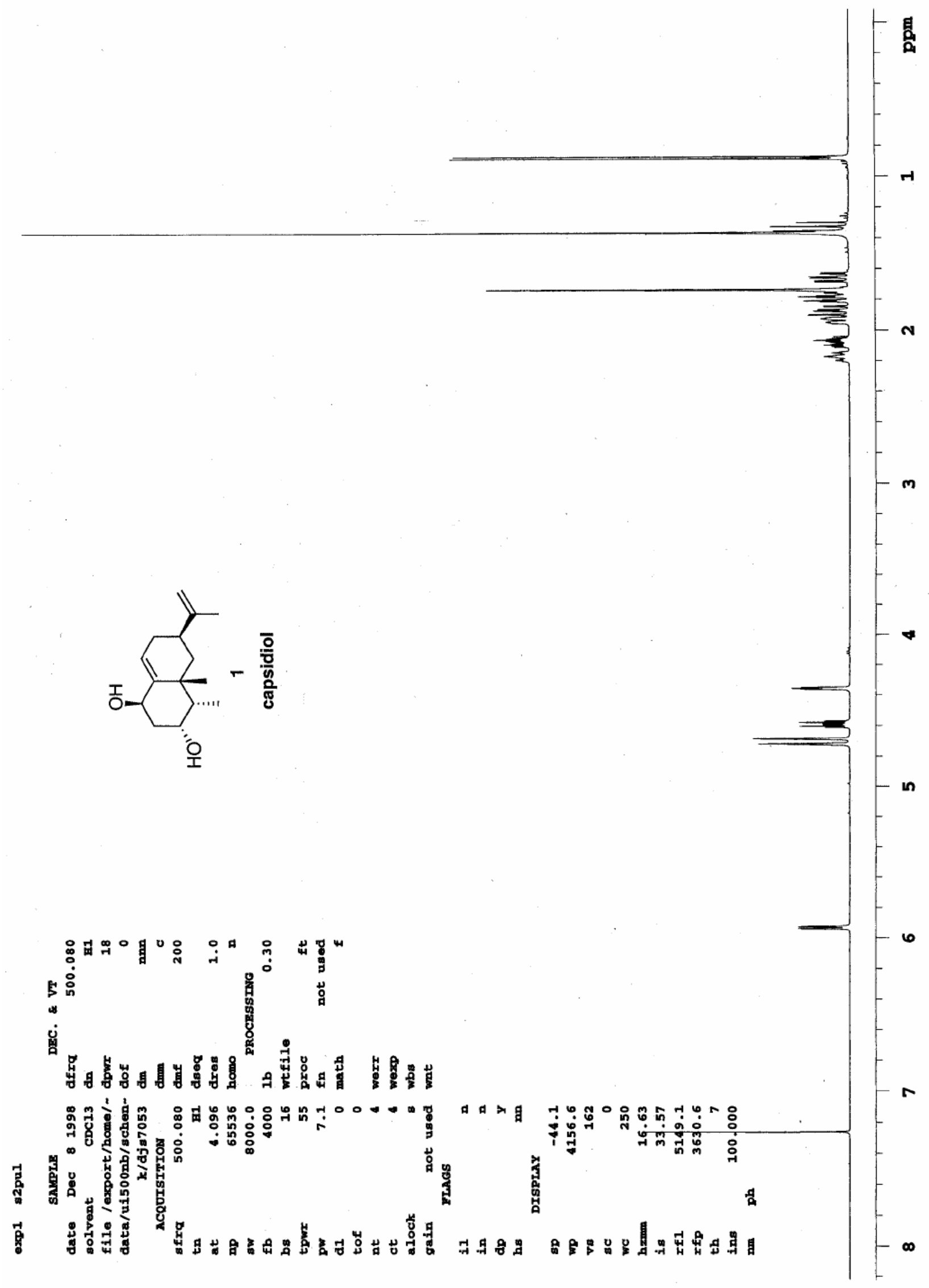




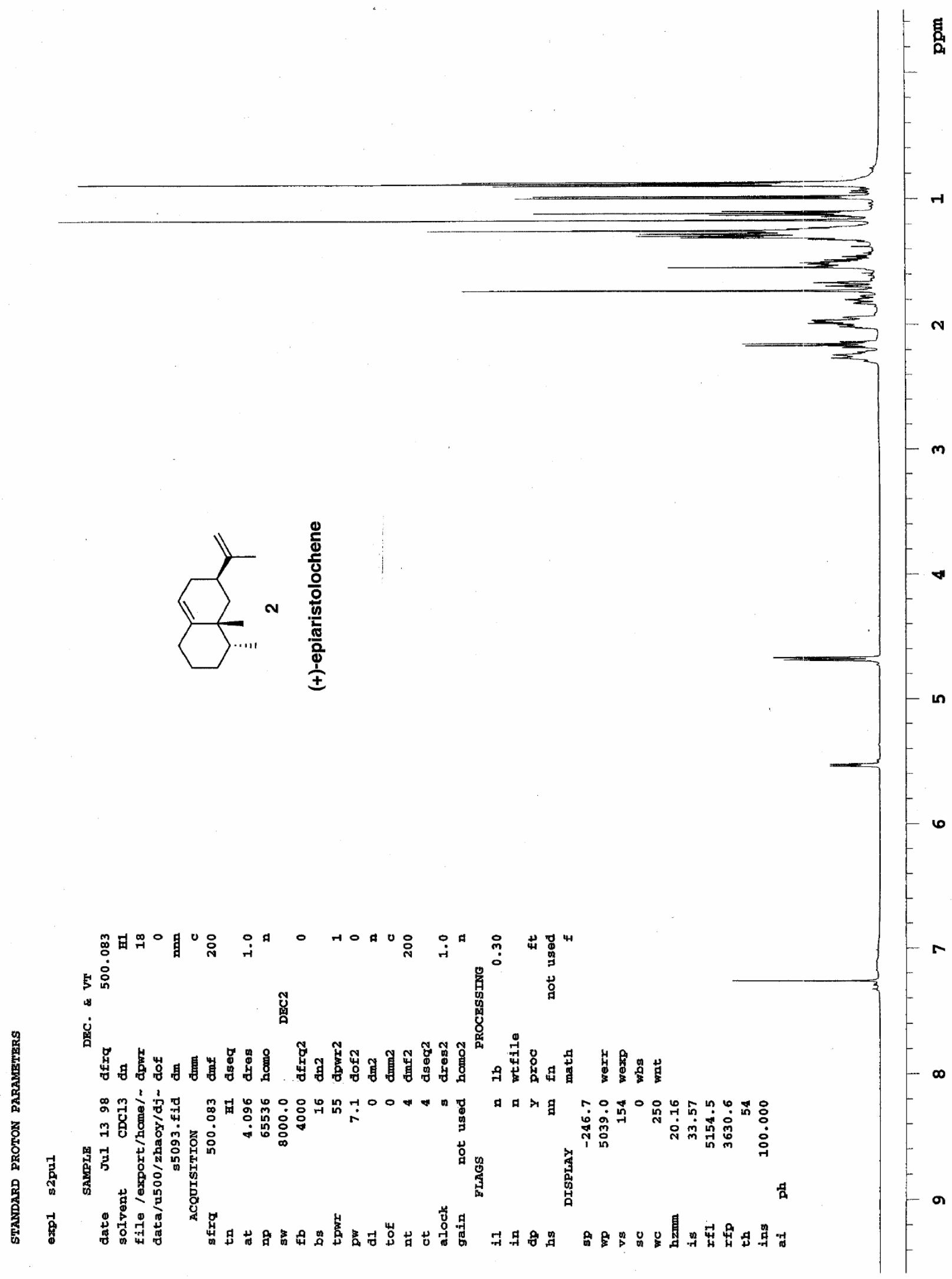




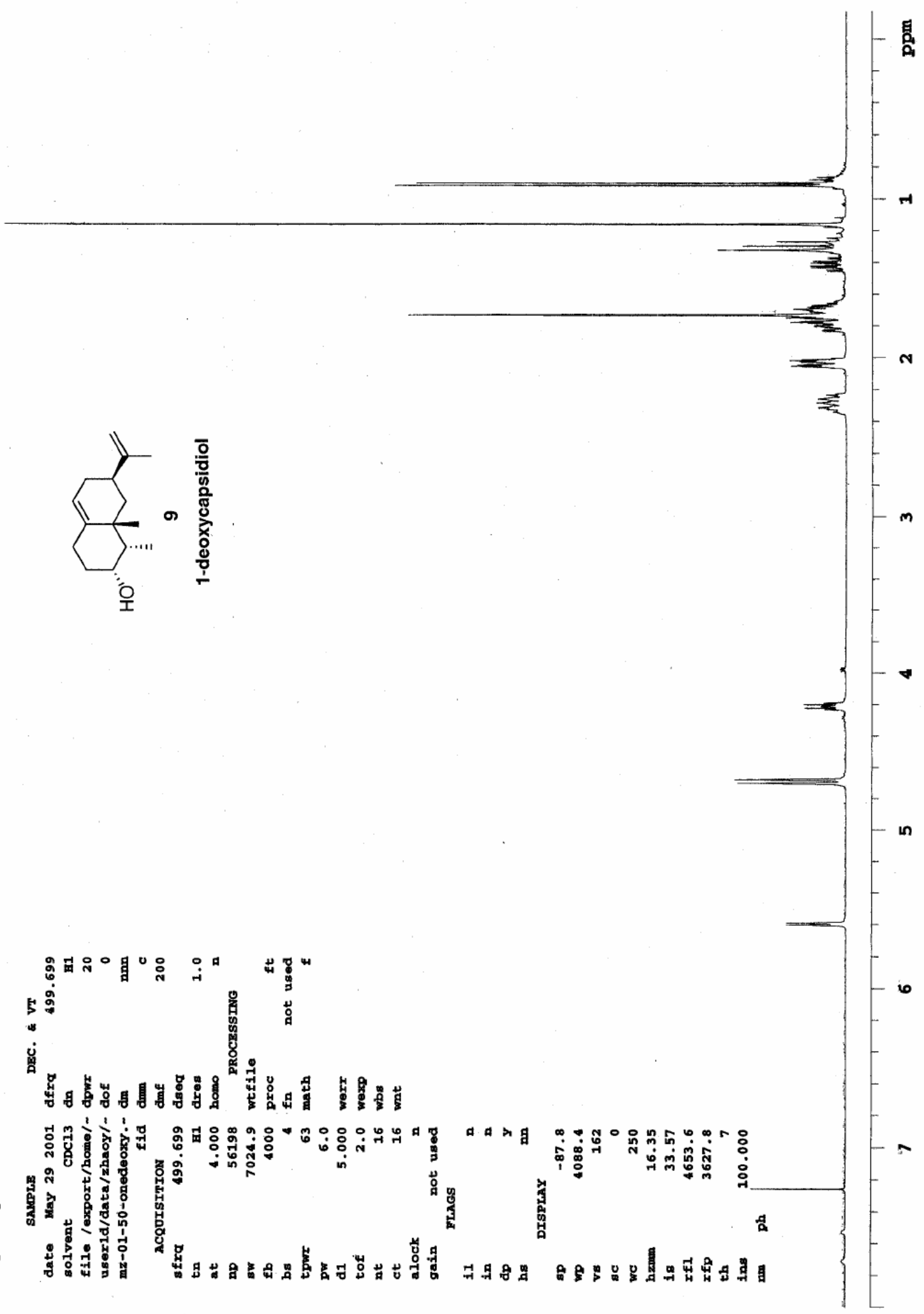




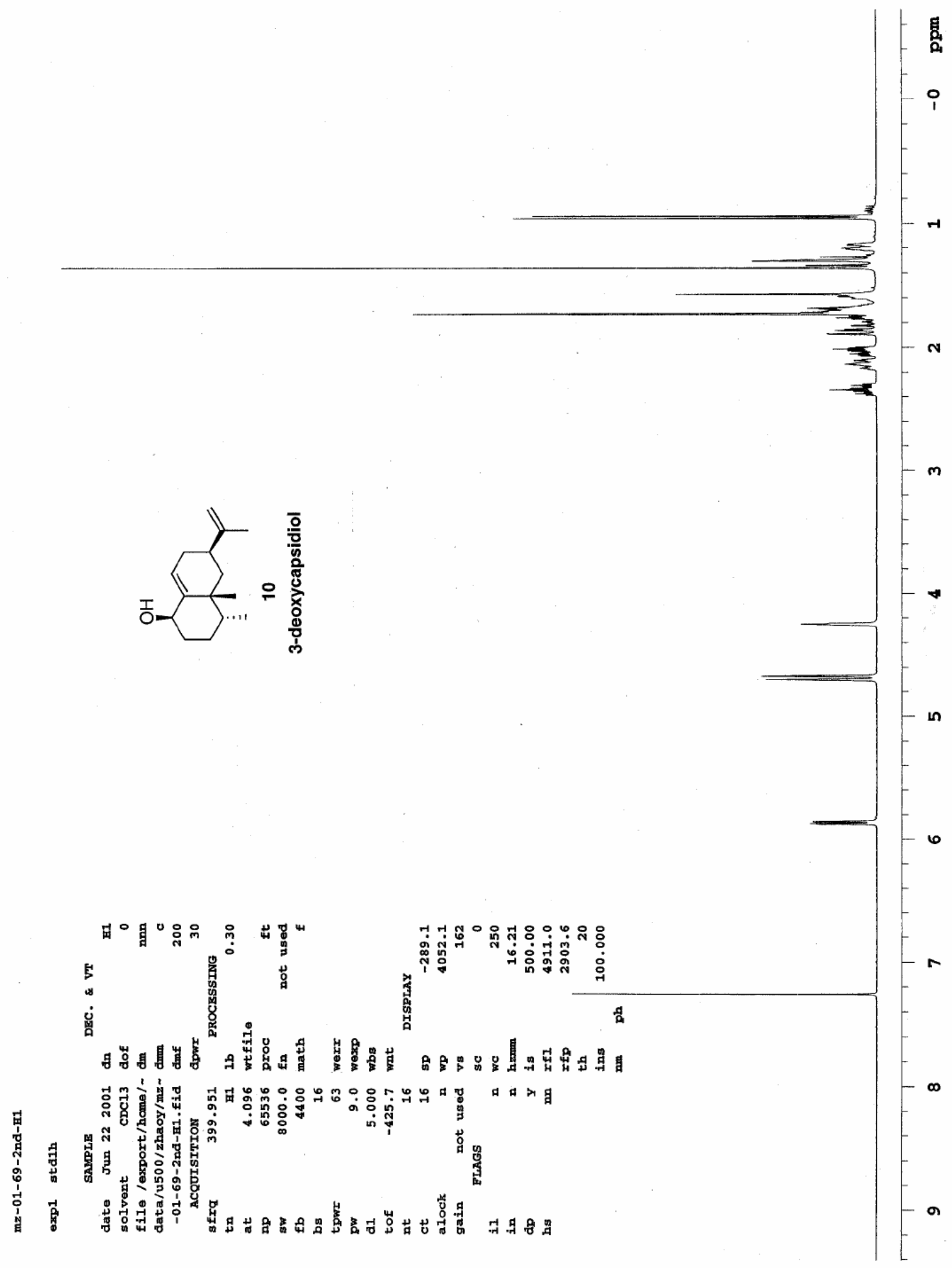




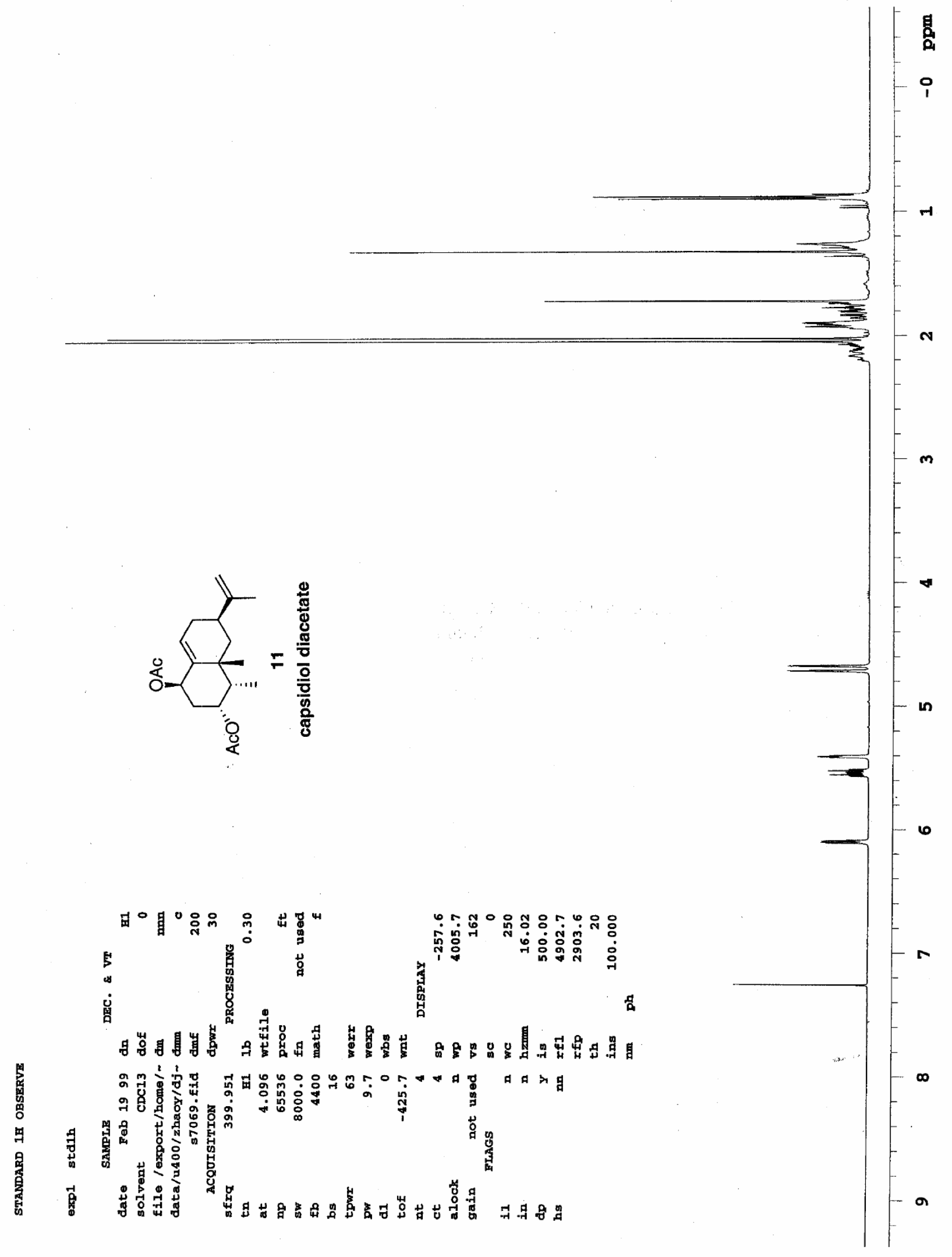




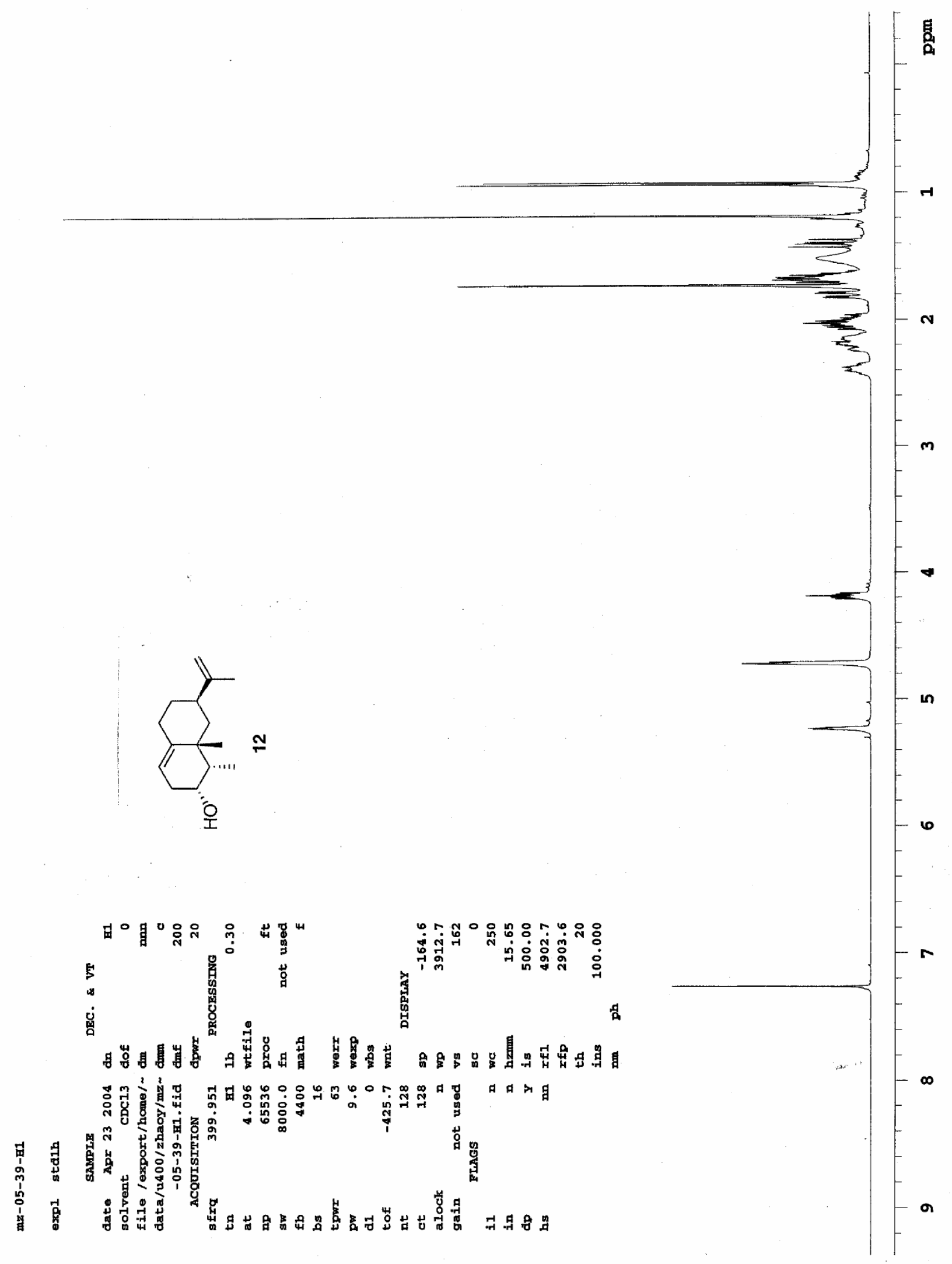




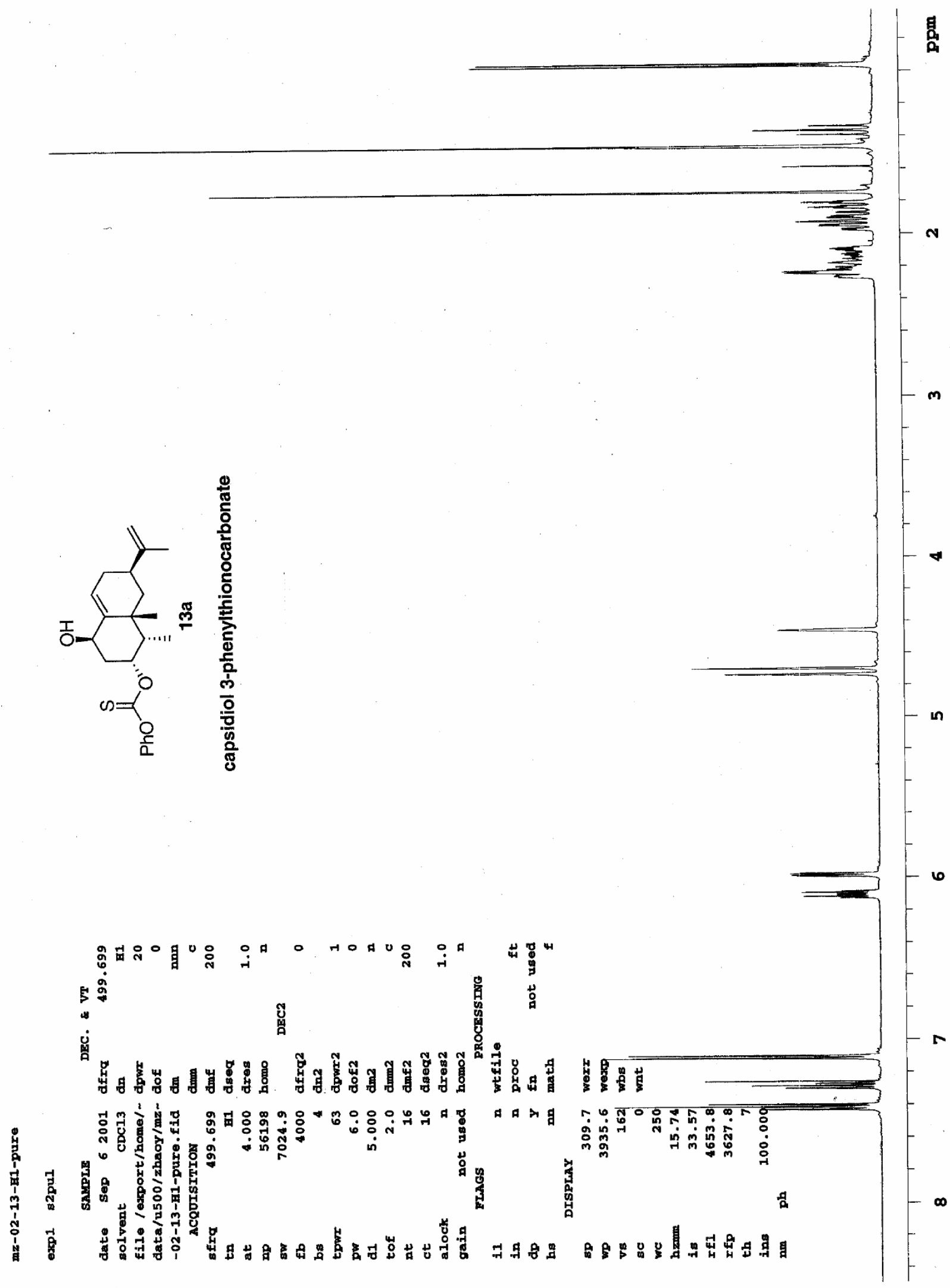




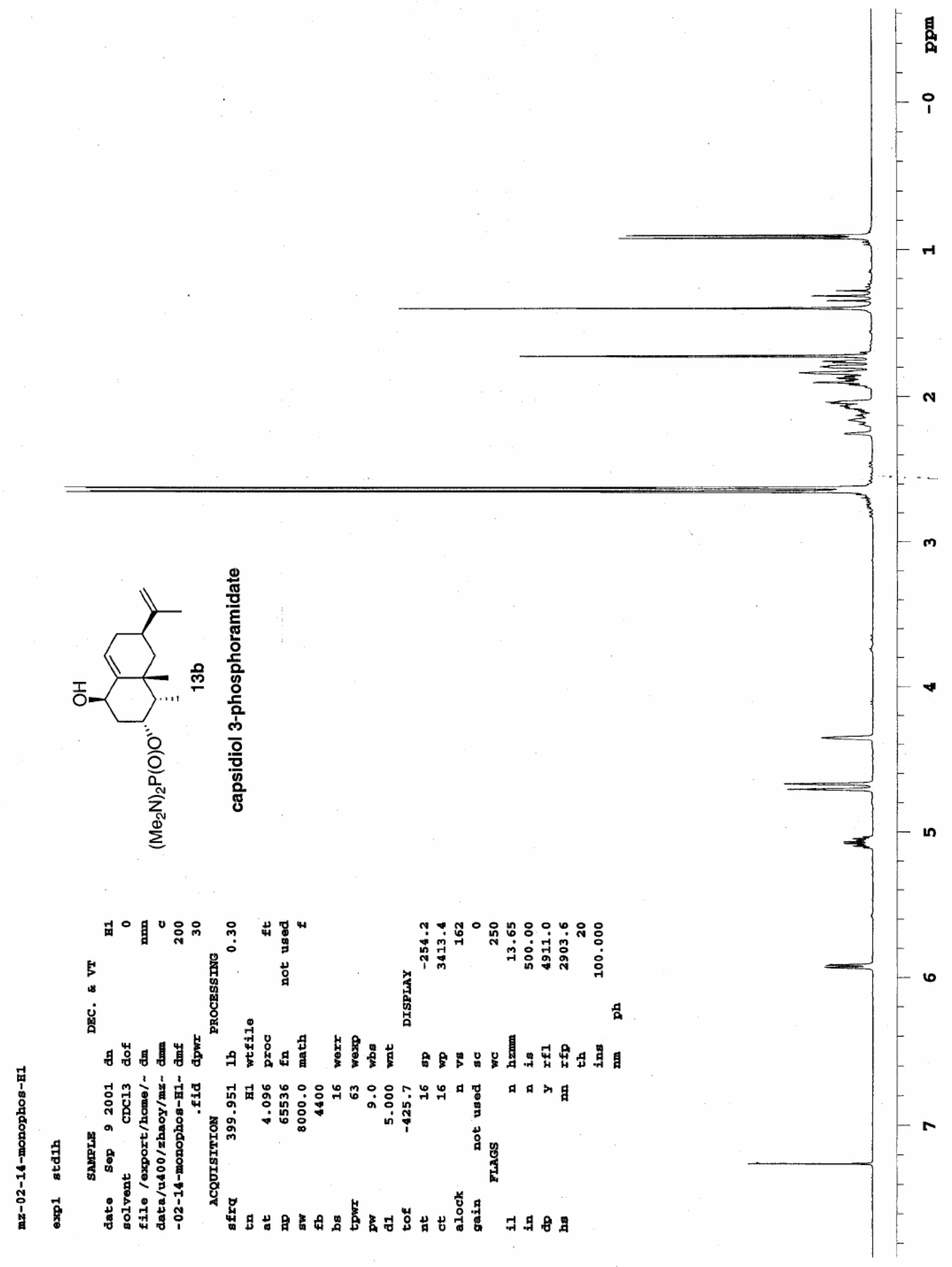




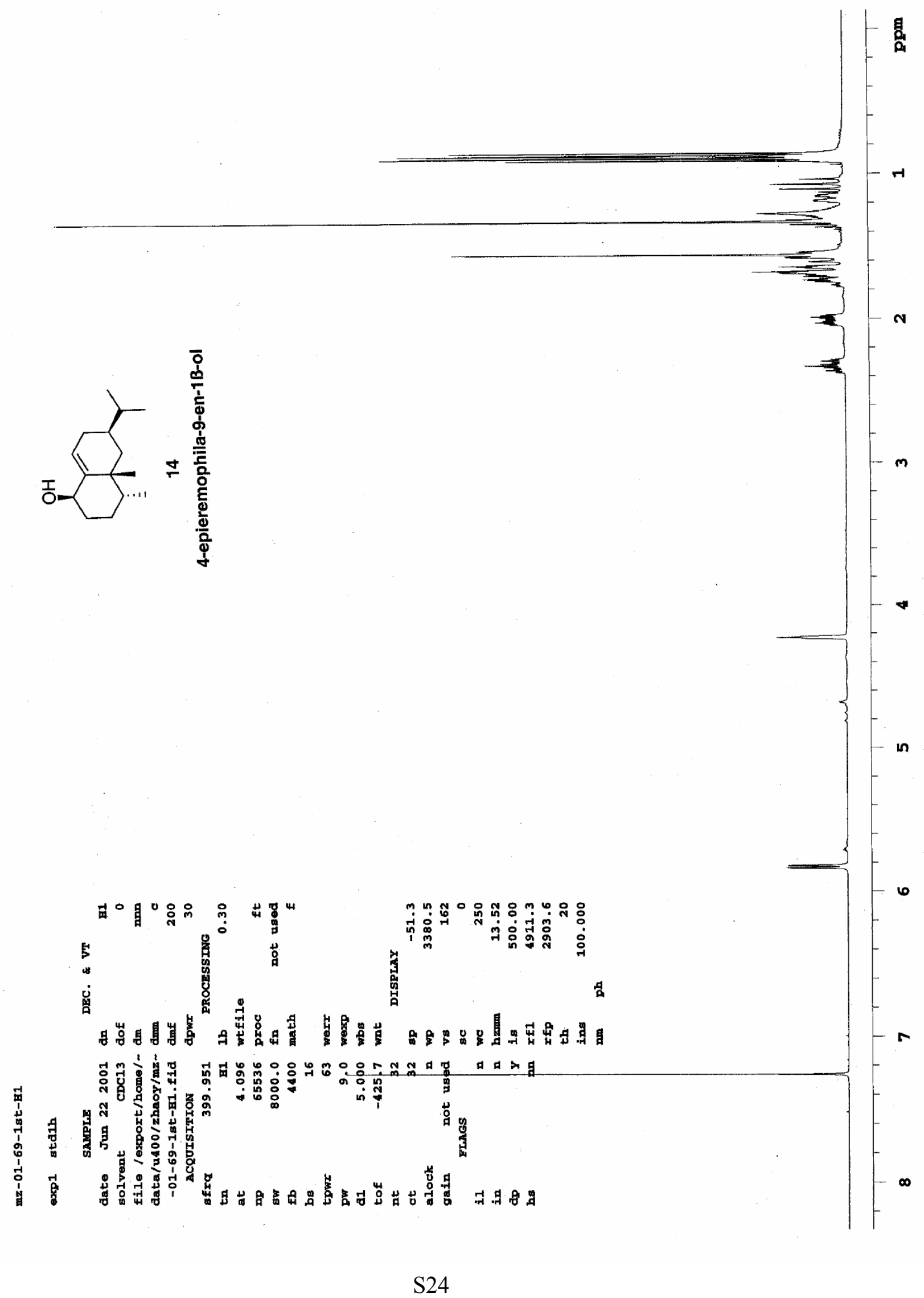




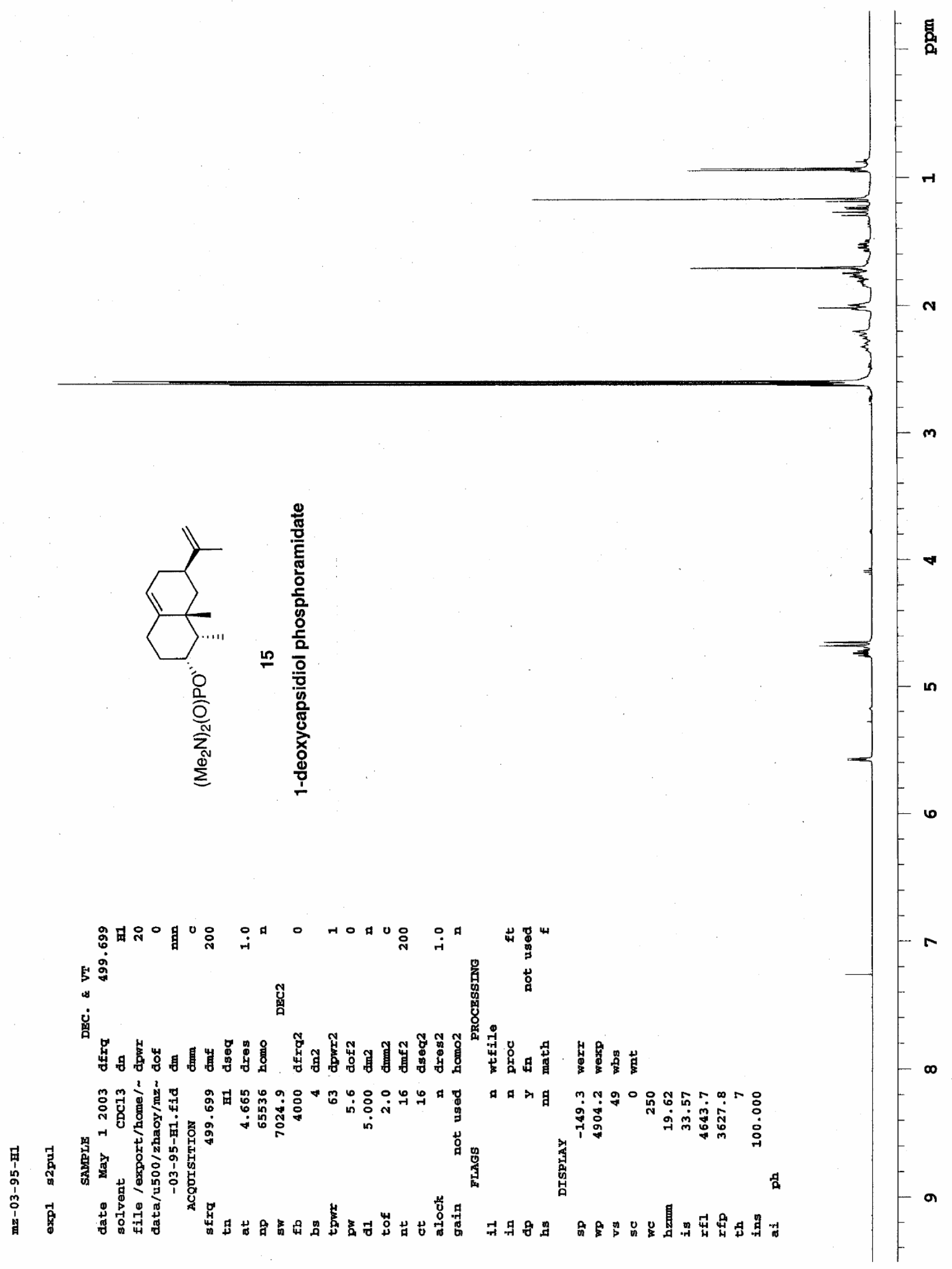




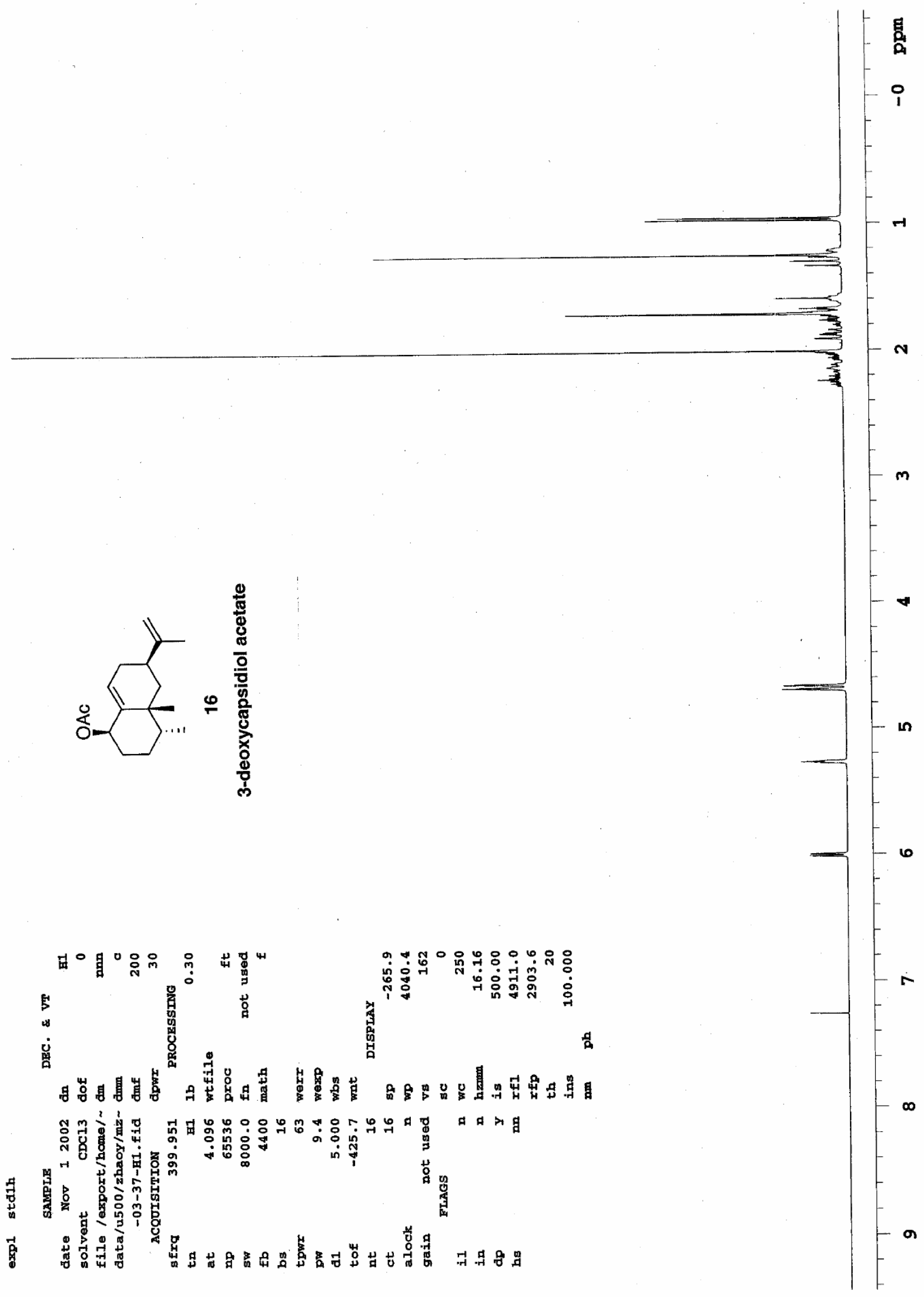




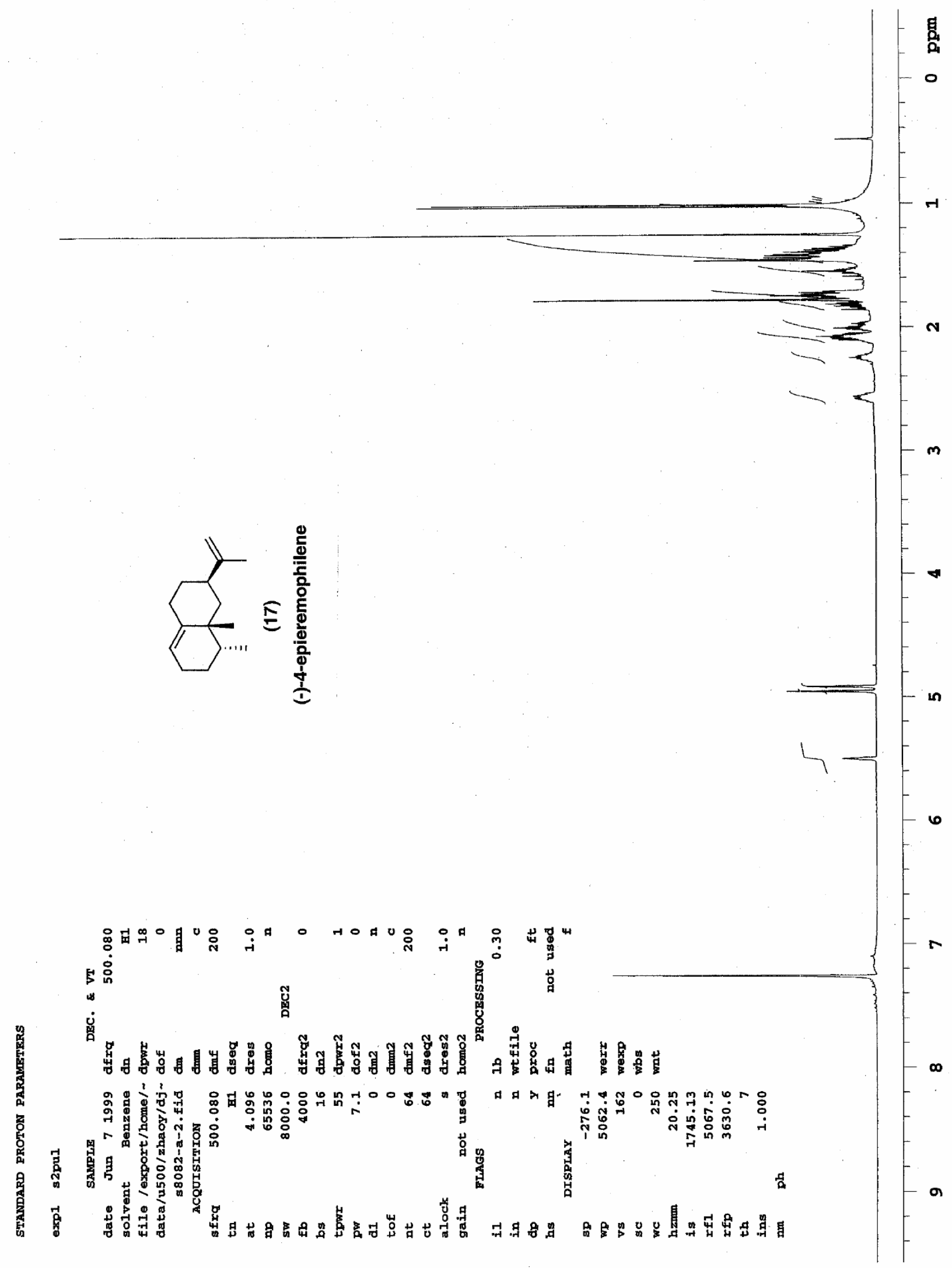




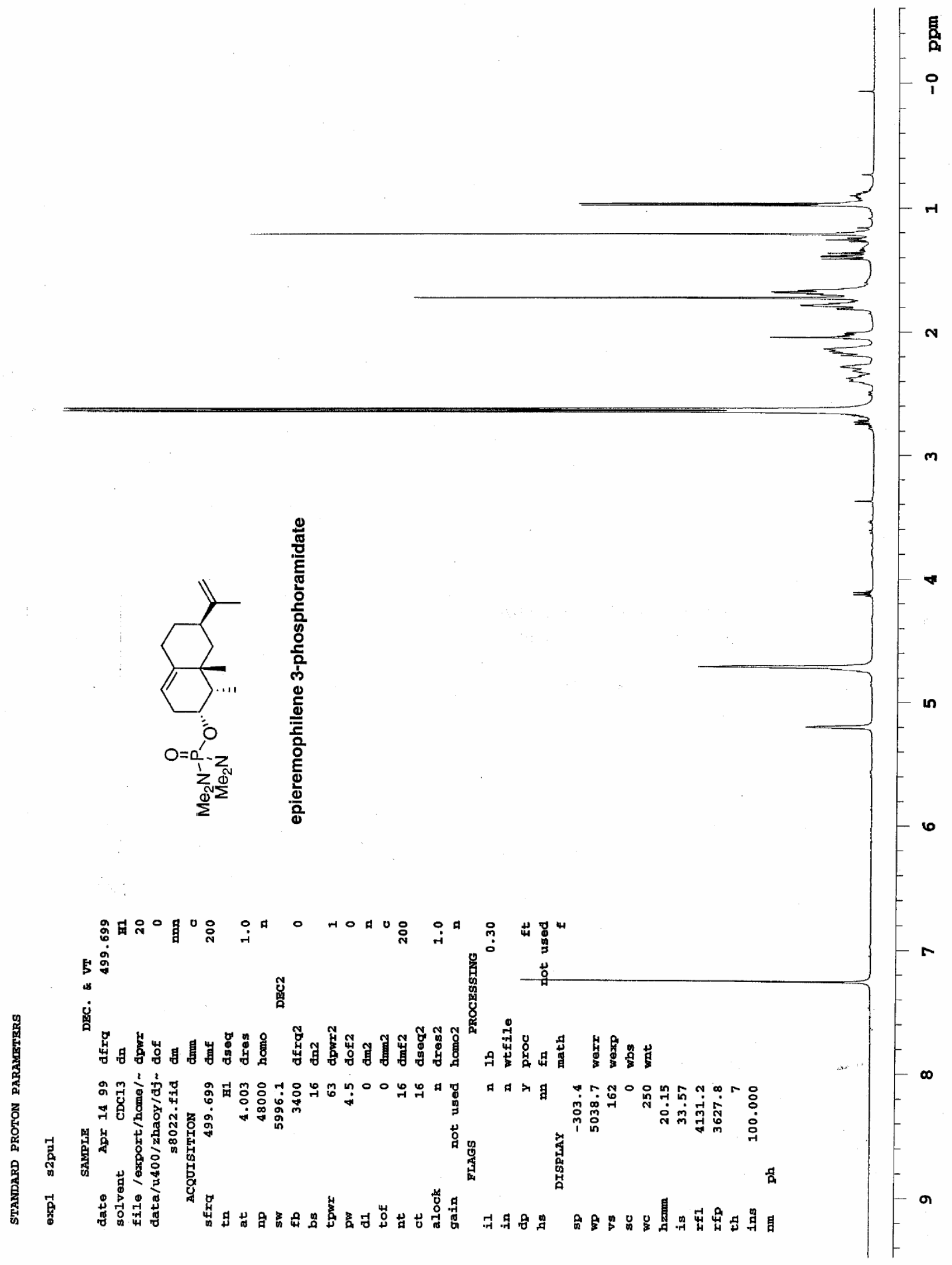




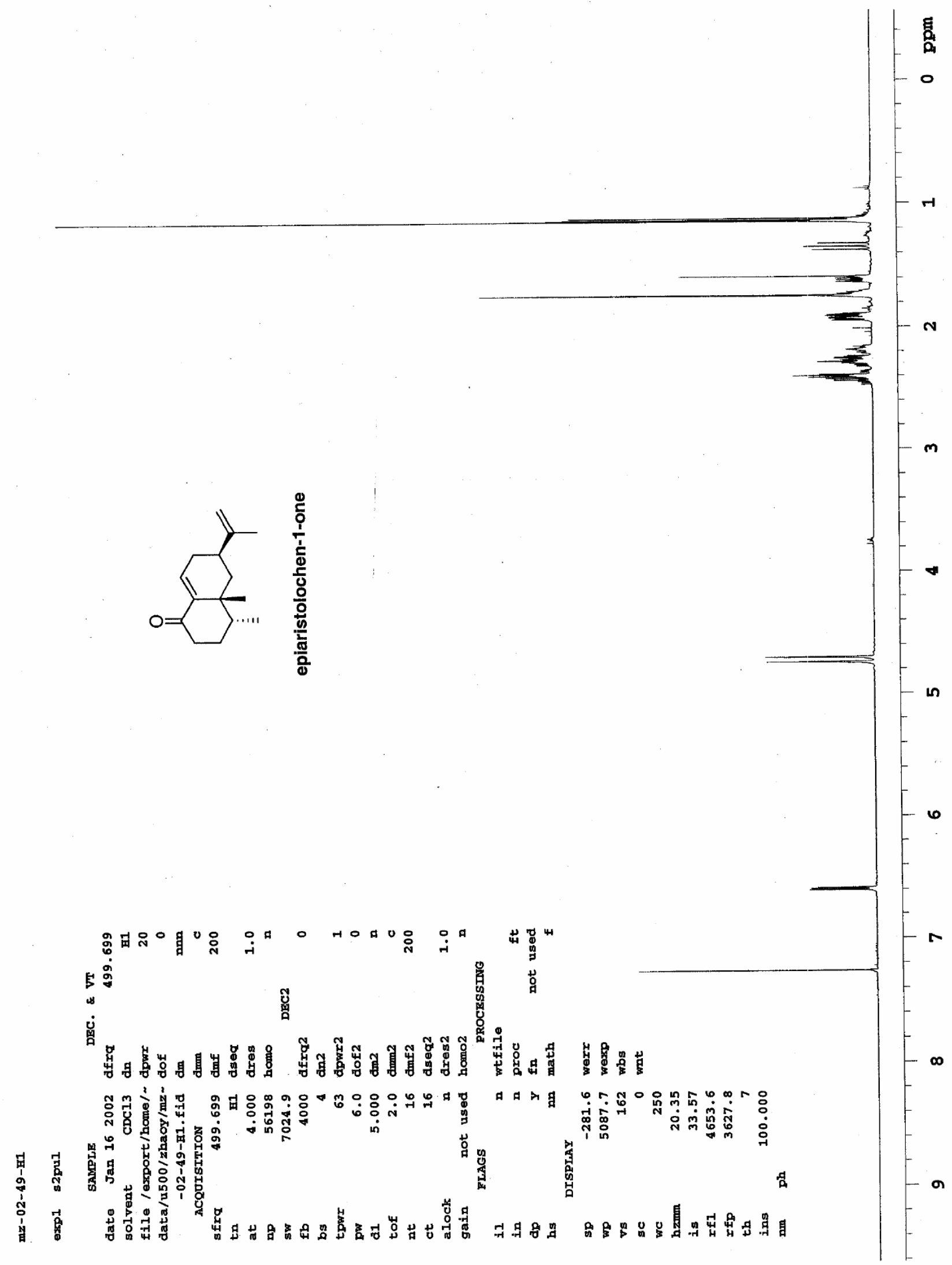




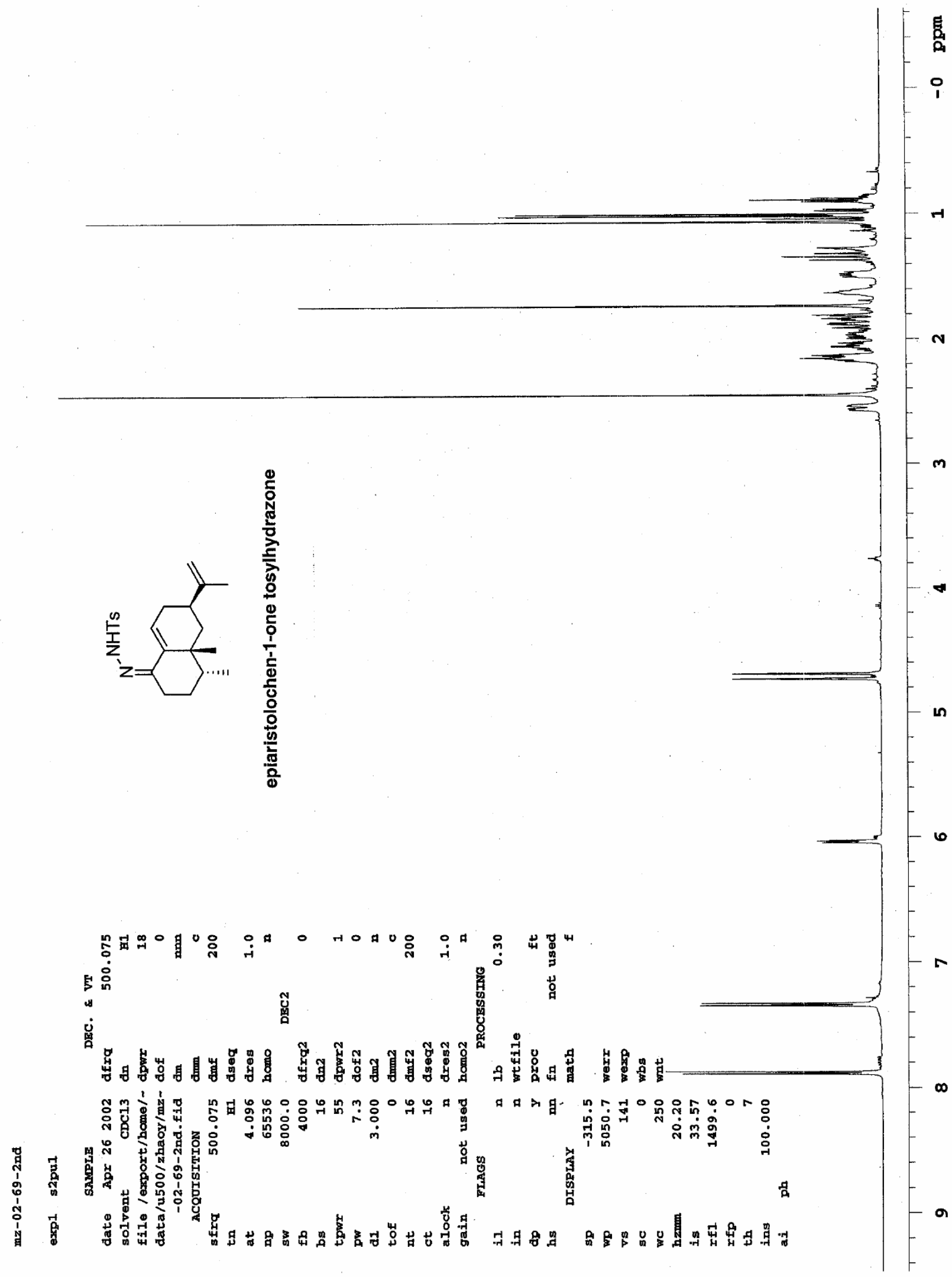




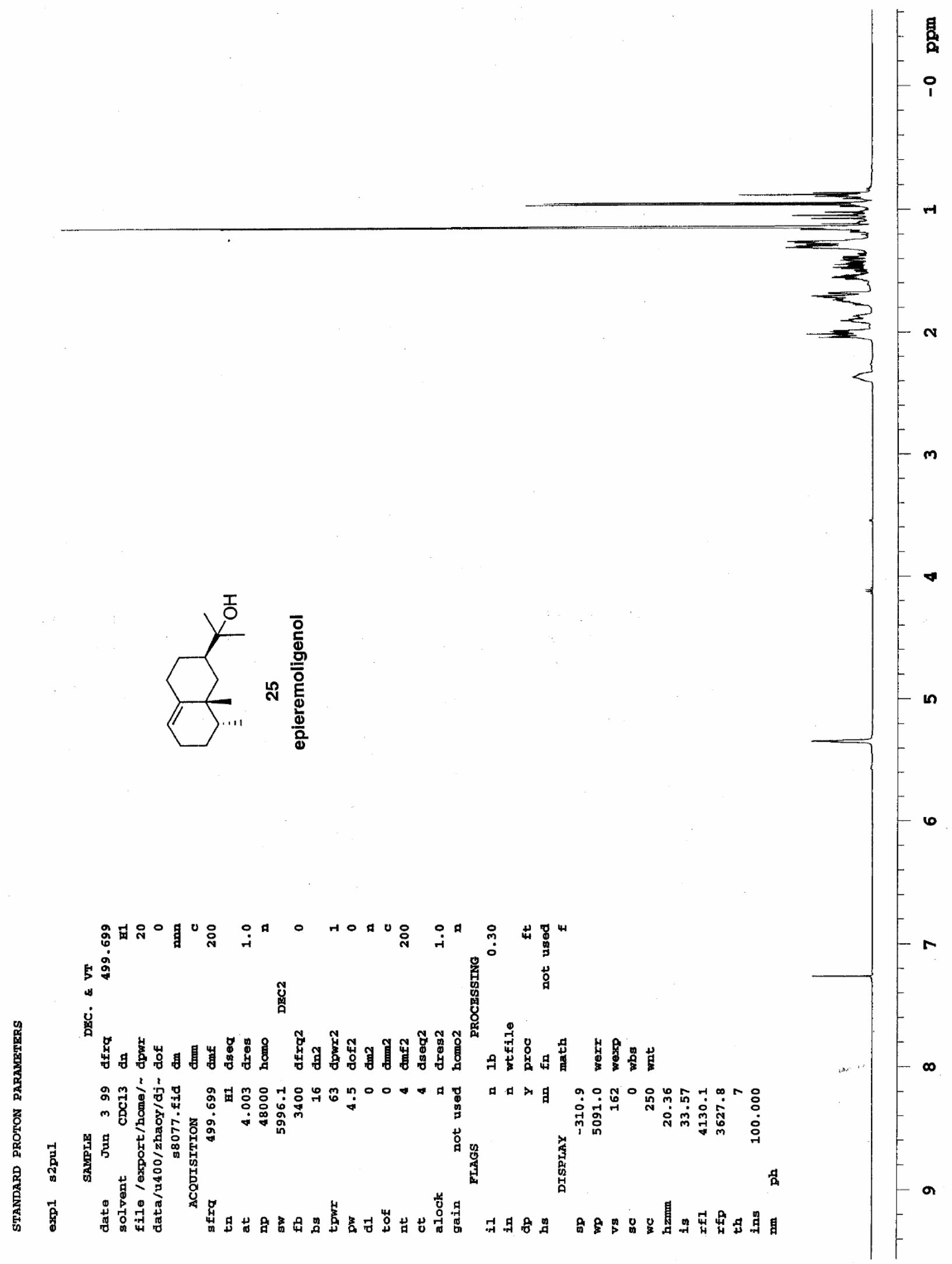




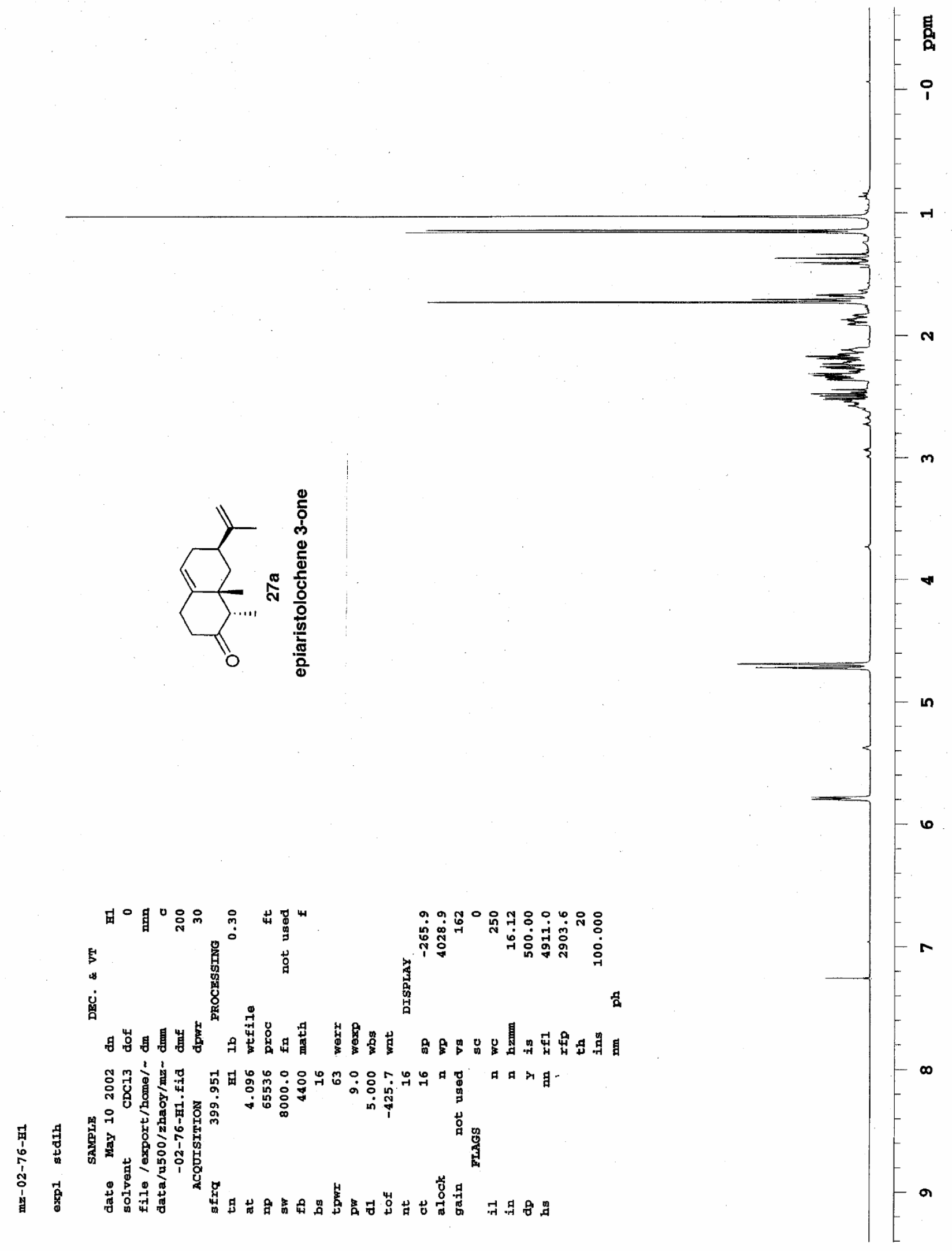




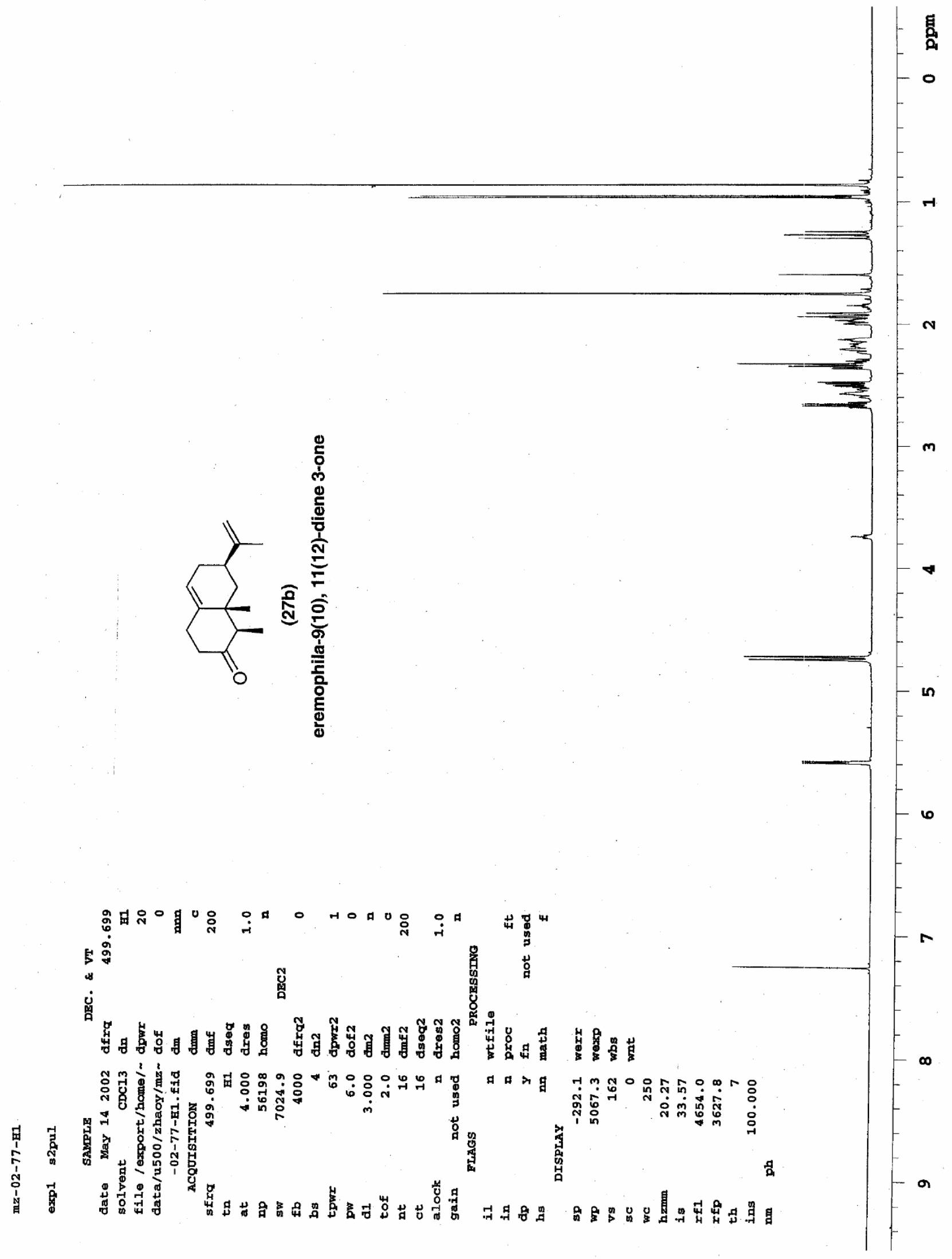




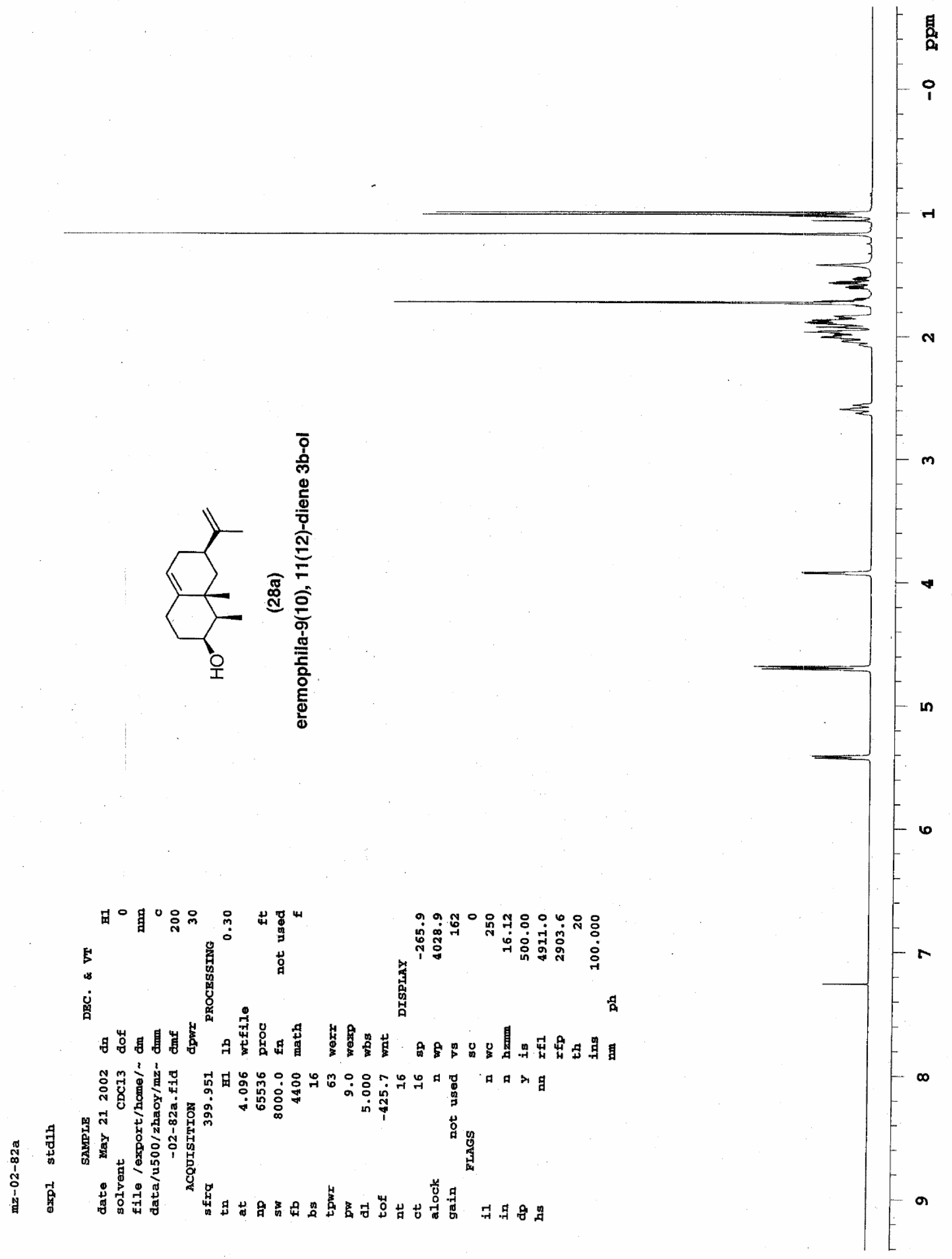




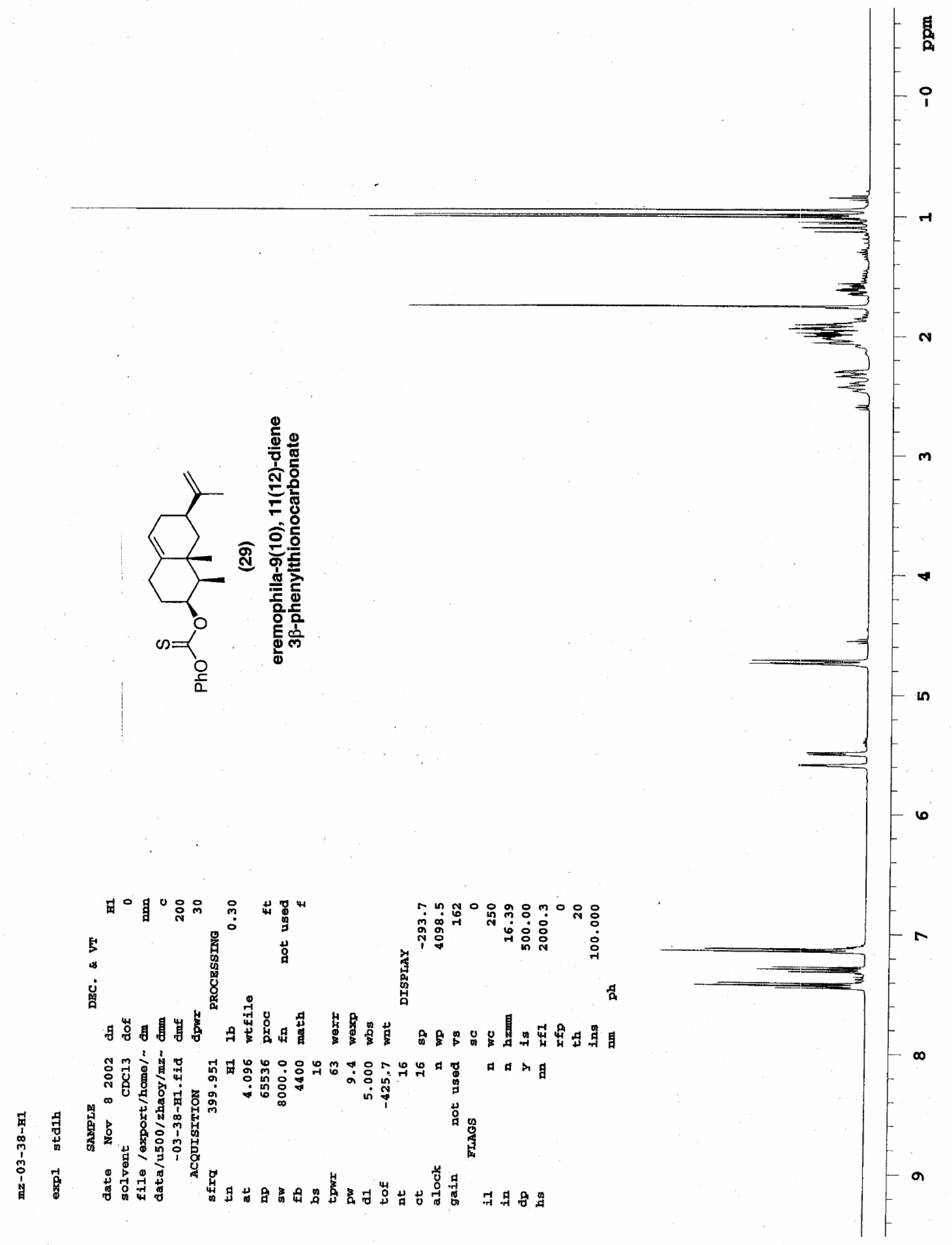




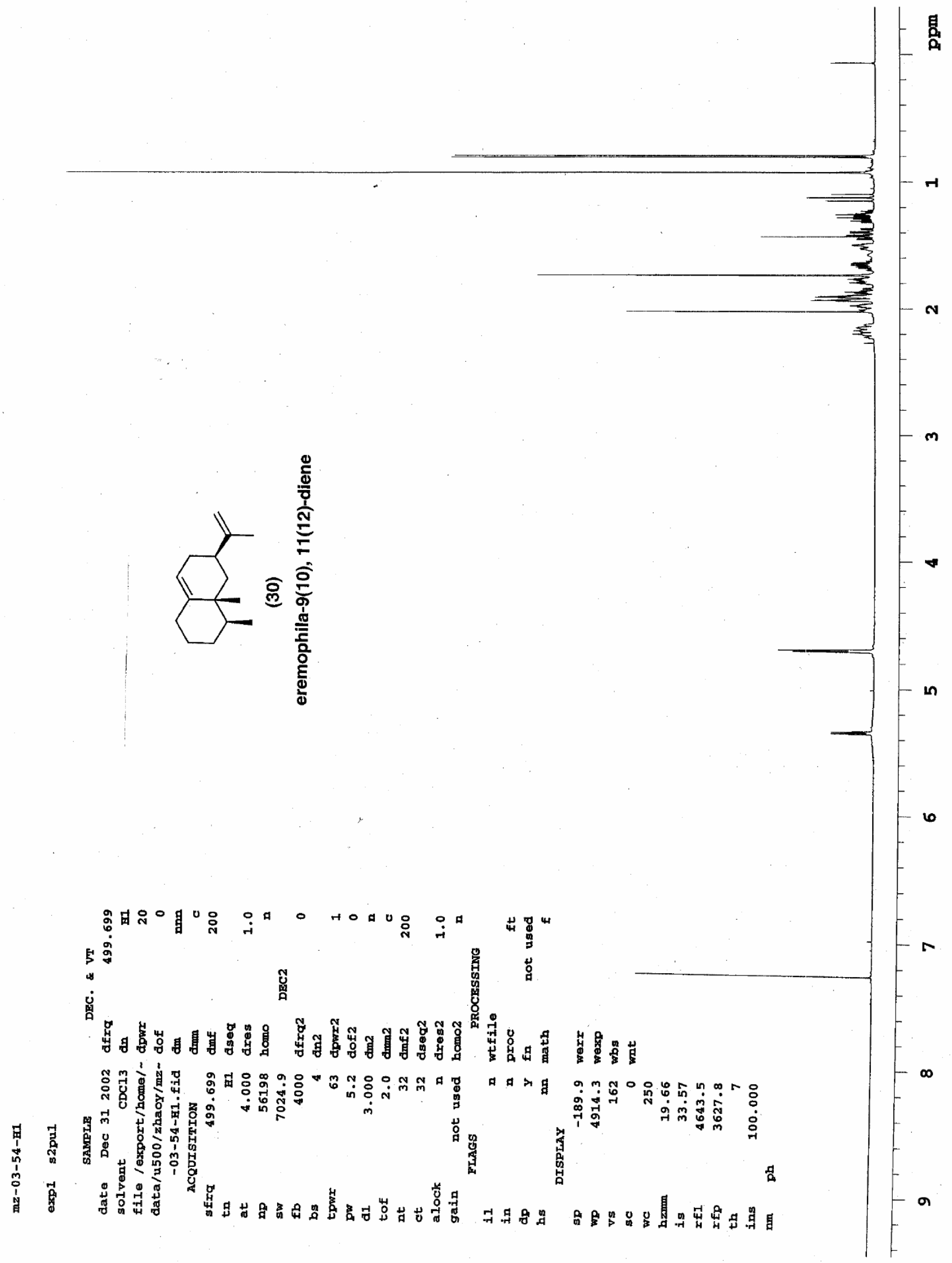




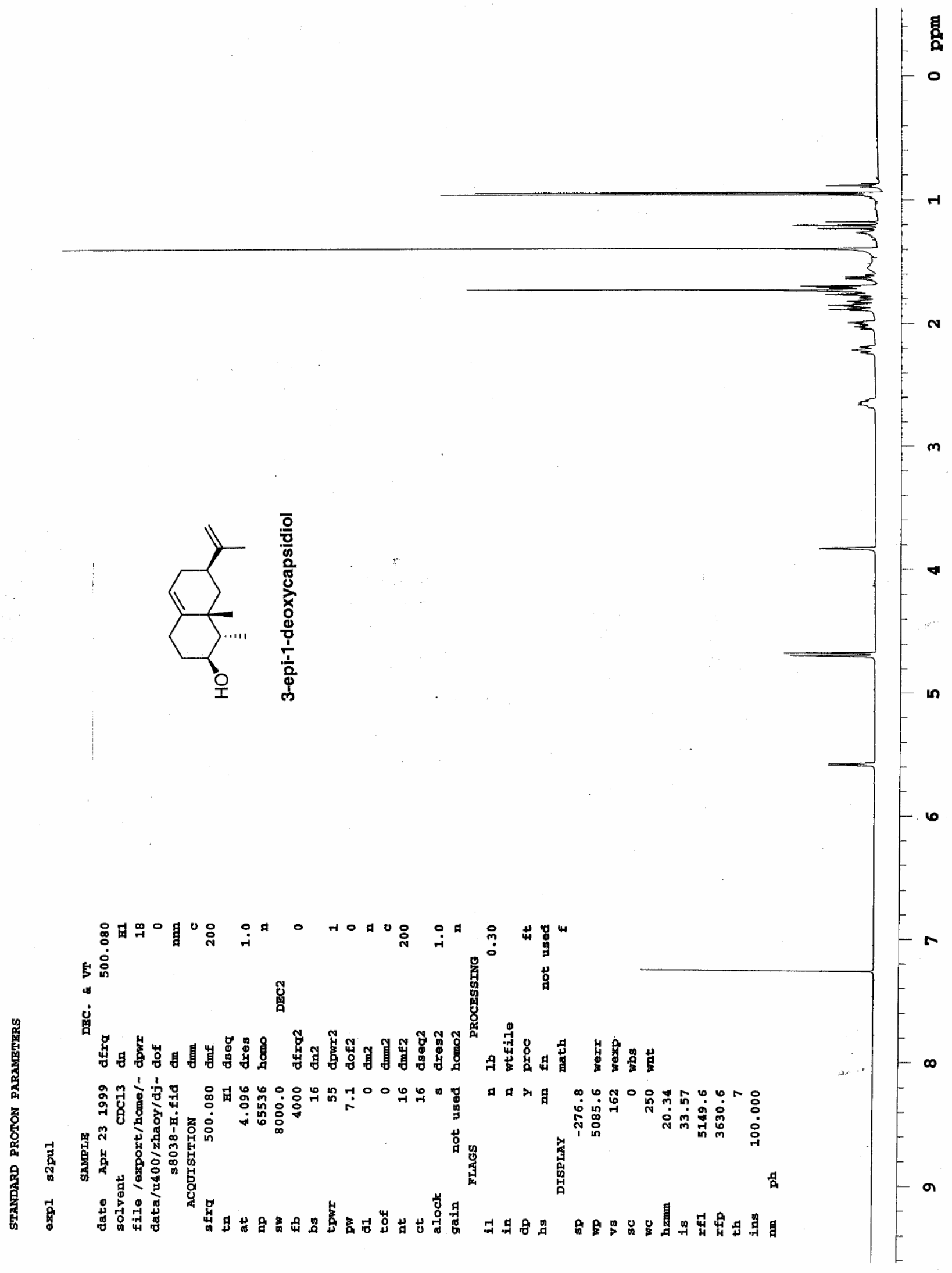

